\title{
Aggregation of a Tetrasaccharide Acceptor Observed by NMR: Synthesis of Pentasaccharide Fragments of the $\operatorname{Le}^{a} \mathrm{Le}^{\mathrm{x}}$ Tumor-Associated Hexasaccharide Antigen
}

\author{
Deng Kuir, Mickaël Guillemineau and France-Isabelle Auzanneau* \\ Department of Chemistry, University of Guelph, Guelph, Ontario, N1G 2WI, Canada \\ Corresponding author. Tel: 01-519-824-4120, ext. 53809; fax: 01-519-766-1499; \\ e-mail: fauzanne@uoguelph.ca \\ Supplementary Material \\ Table of Contents
}

General Experimental Procedures..................................2

${ }^{1} \mathrm{H}$ - and ${ }^{13} \mathrm{C}$ - NMR spectra for all new compounds..................... 


\section{General Experimental Procedures}

${ }^{1} \mathrm{H}$ NMR (400 and $600 \mathrm{MHz}$ ) and ${ }^{13} \mathrm{C}$ NMR (100.6 or 150.9) spectra were recorded with Bruker Avance spectrometers in CDCl $\mathrm{C}_{3}$ (internal standard, for ${ }^{1} \mathrm{H}$ residual $\mathrm{CHCl}_{3} \delta_{\mathrm{H}} 7.24$; for ${ }^{13} \mathrm{C} \mathrm{CDCl}_{3}, \delta_{\mathrm{C}} 77.0$ ), $\mathrm{CD}_{3} \mathrm{COD}$ (internal standard, for ${ }^{1} \mathrm{H}$ residual $\mathrm{CD}_{2} \mathrm{HOD} \delta_{\mathrm{H}} 3.30$; for ${ }^{13} \mathrm{C} \delta_{\mathrm{C}} 49.0$ ) or $\mathrm{D}_{2} \mathrm{O}$ [external standard (3-(trimethylsilyl)-propionic acid-d ${ }_{4}$, sodium salt (TSP) for $\delta_{\mathrm{H}}$ 0.00; for $\left.{ }^{13} \mathrm{C} \delta_{\mathrm{C}} 0.0\right)$. ${ }^{1} \mathrm{H}$ NMR and ${ }^{13} \mathrm{C}$ NMR chemical shifts are reported in part per million (ppm). Coupling constants $(J)$ were obtained from a first-order analysis of one-dimensional spectra and are reported in Hertz (Hz). Assignments of proton and carbon resonances were based on two dimensional ${ }^{1} \mathrm{H}-{ }^{1} \mathrm{H}$ COSY and ${ }^{13} \mathrm{C}-{ }^{1} \mathrm{H}$ HSQC correlation experiments. ${ }^{1} \mathrm{H}$ NMR data are reported using standard abbreviations: singlet (s) doublet (d), triplet (t), doublet of doublet (dd), double of doublet of doublet (ddd), triplet of doublet (td), broad signal (br, s) and multiplet (m). Analytical thin-layer chromatography (TLC) was performed on aluminium plates pre-coated with Silica Gel 60 F234 $(250 \mu \mathrm{m})$ containing a fluorescent indicator. The plates were visualized under UV and/or charred with $20 \%$ solution of $\mathrm{H}_{2} \mathrm{SO}_{4}$ in EtOH. Compounds were purified by flash chromatography with Silica Gel 60 (230-400 mesh) unless otherwise stated. Solvents were distilled and dried according to standard procedures and organic solutions were dried over $\mathrm{Na}_{2} \mathrm{SO}_{4}$ and concentrated under reduced pressure. All reagents were purchased from commercial suppliers and used without further purification. Molecular sieves were activated by heating at $300^{\circ} \mathrm{C}$ overnight under reduced pressure. Zinc powder ( $2 \mathrm{~g}$ ) was activated by successive washings with $1 \mathrm{~N} \mathrm{HCl}(25 \mathrm{~mL}), \mathrm{H}_{2} \mathrm{O}(50 \mathrm{~mL}), \mathrm{MeOH}(50 \mathrm{~mL})$, and $\mathrm{Et}_{2} \mathrm{O}(50 \mathrm{~mL})$ on a fritted glass. Sonication reactions were carried out in a thermo-controlled $\left(50 \pm 4{ }^{\circ} \mathrm{C}\right)$ ultrasonic cleaning water bath at $40 \mathrm{kHz}$. RP-HPLC purifications were carried out on Prep Nova Pak ${ }^{\circledR}$ HR C18, $6 \mu \mathrm{m} 60 \AA$ columns using a mixture of HPLC grade acetonitrile and milli-Q water. Optical rotations were measured at $22{ }^{\circ} \mathrm{C}$ and are reported as follows: $[\alpha]_{\mathrm{D}}[c$ in $\mathrm{g}$ per $100 \mathrm{~mL}$ of solvent]. Purifications with gel permeation chromatography were carried out on a Biogel P2 column $(95 \mathrm{~cm} \times 1.5 \mathrm{~cm})$ eluted with Milli-Q water or with a $0.05 \mathrm{M}$ aqueous ammonium acetate solution. 
Compound 11, $400 \mathrm{MHz}, 296 \mathrm{~K}, \mathrm{CDCl}_{3}$

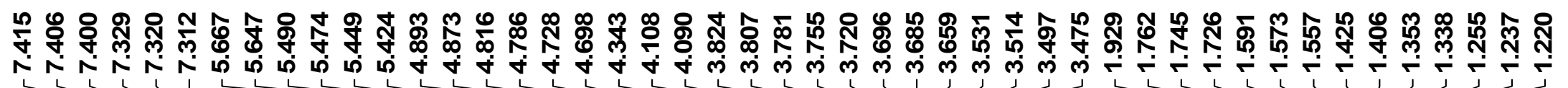
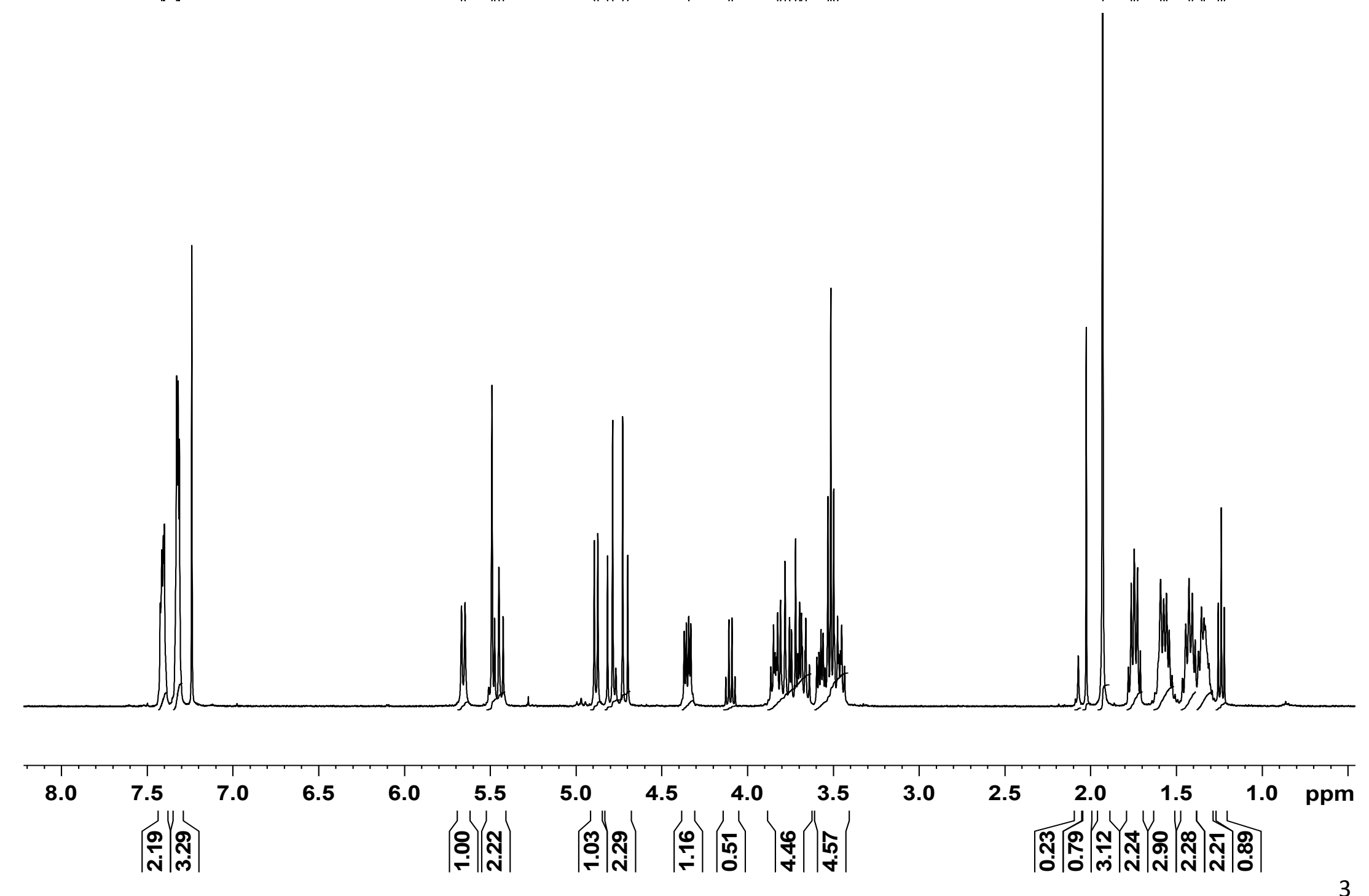
Compound 11, $101 \mathrm{MHz}, 296 \mathrm{~K}, \mathrm{CDCl}_{3}$

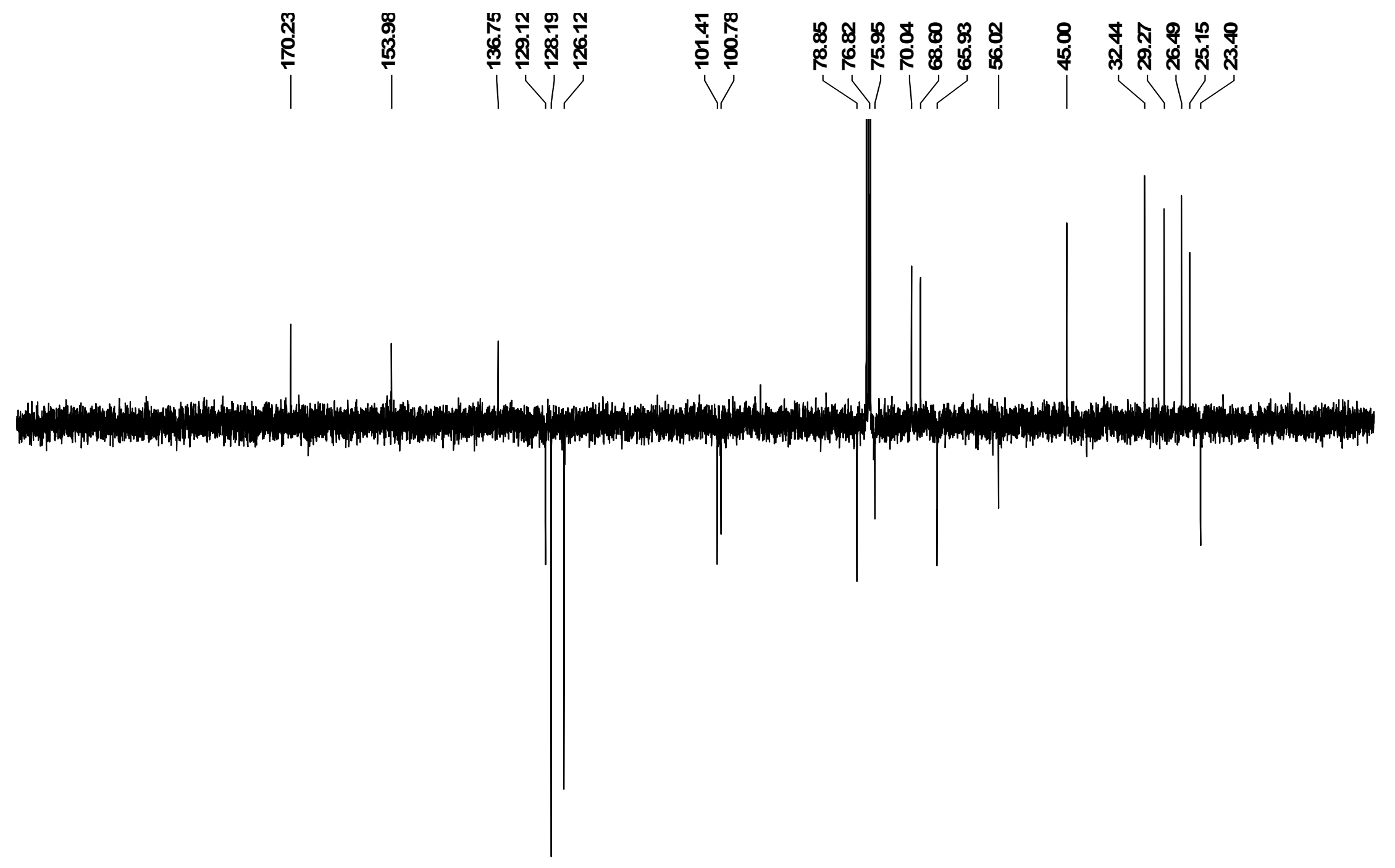

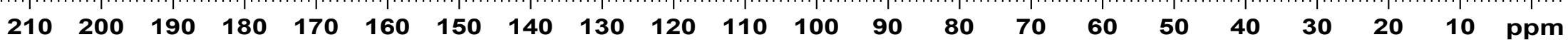




\section{Compound 5, $400 \mathrm{MHz}, 295 \mathrm{~K}, \mathrm{CDCl}_{3}$}

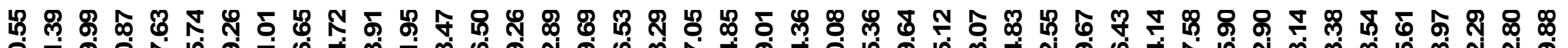

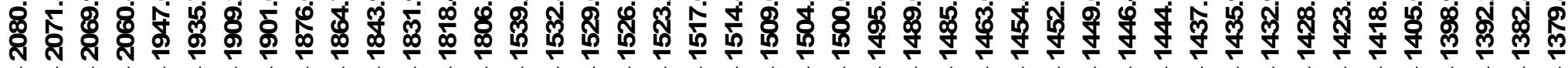

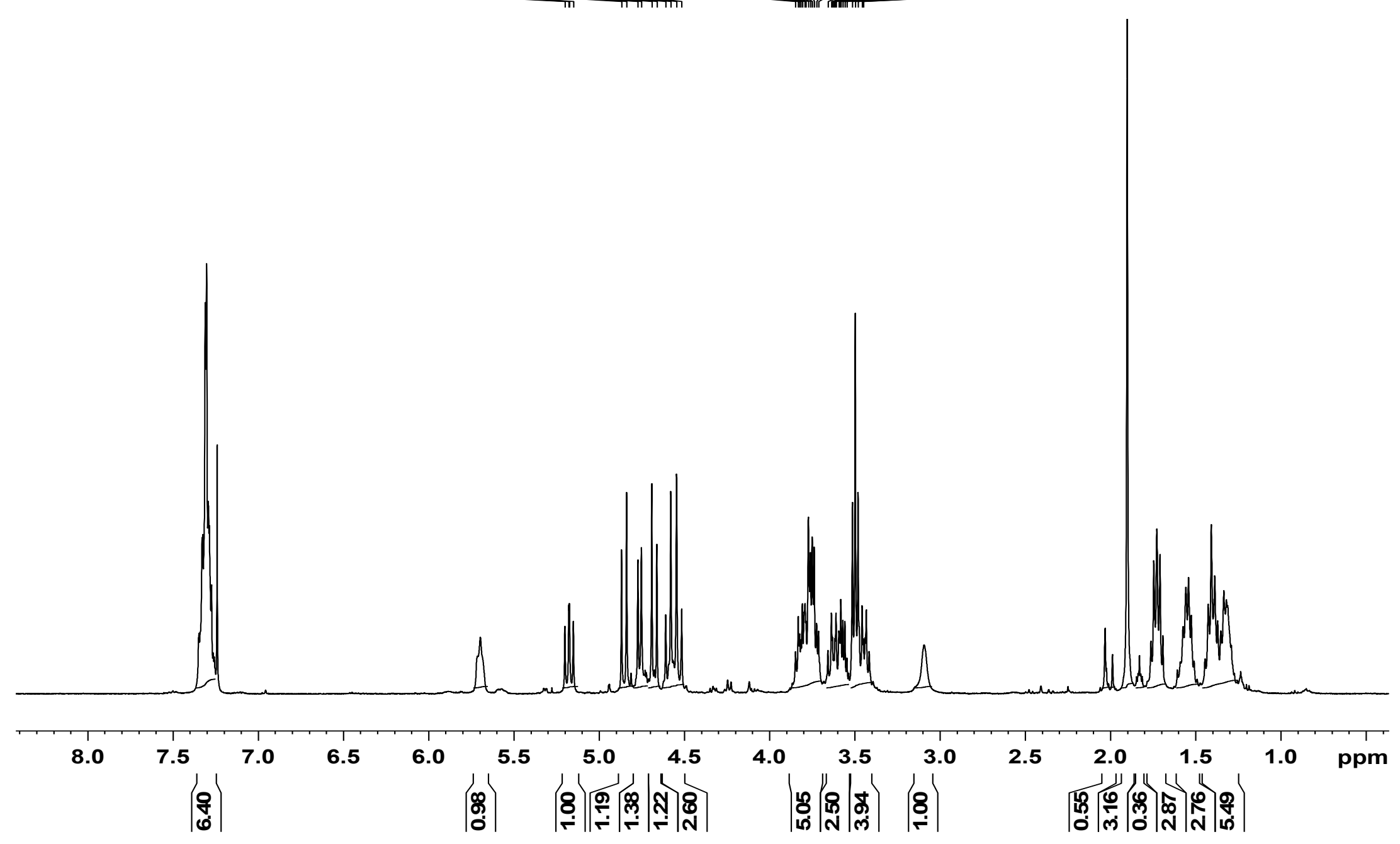


Compound 5, $101 \mathrm{MHz}, 297 \mathrm{~K}, \mathrm{CDCl}_{3}$

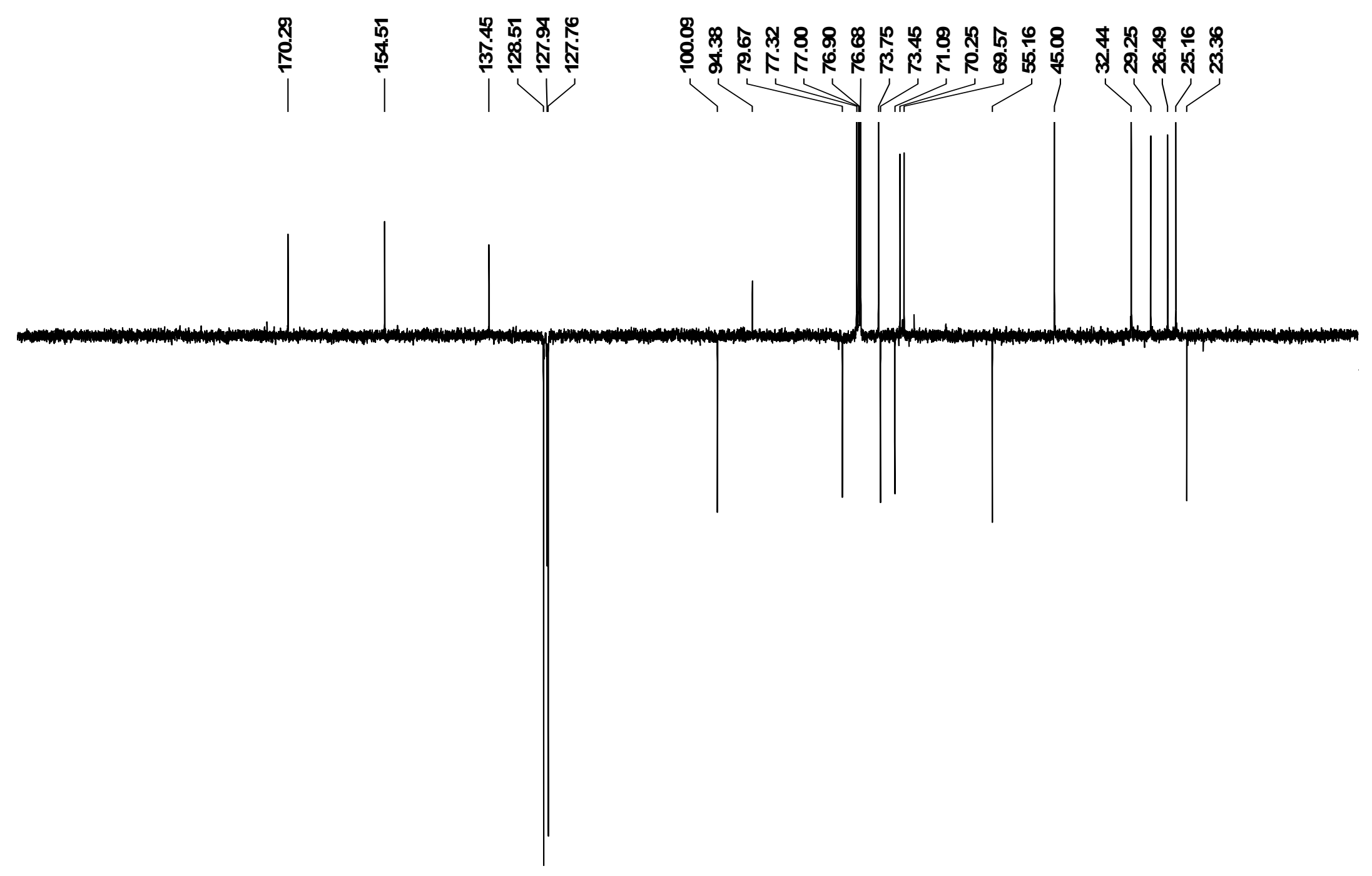


Compound 13, $400 \mathrm{MHz}, 295 \mathrm{~K}, \mathrm{CDCl}_{3}$
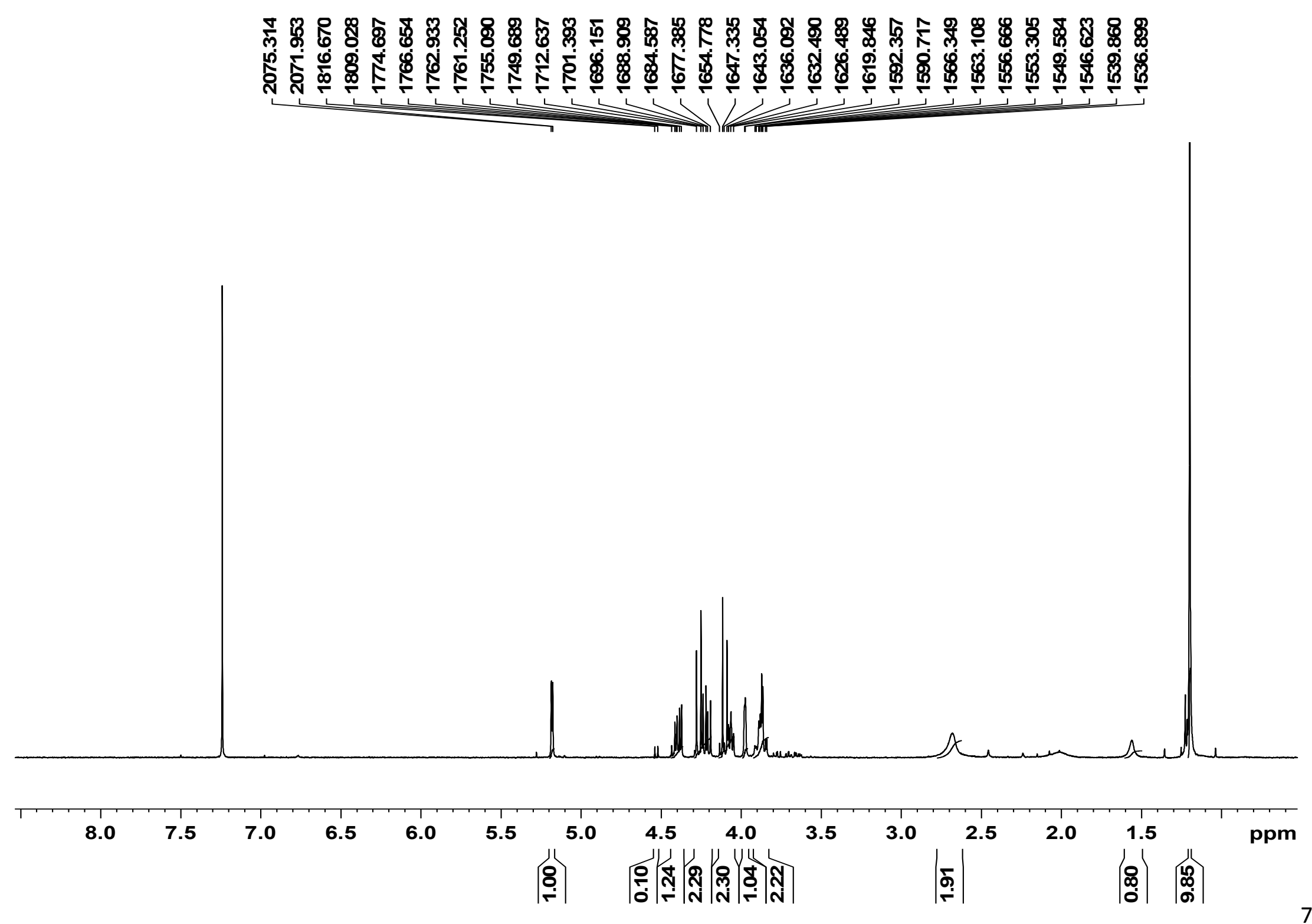


\section{Compound 13, $101 \mathrm{MHz}, 297 \mathrm{~K}, \mathrm{CDCl}_{3}$}

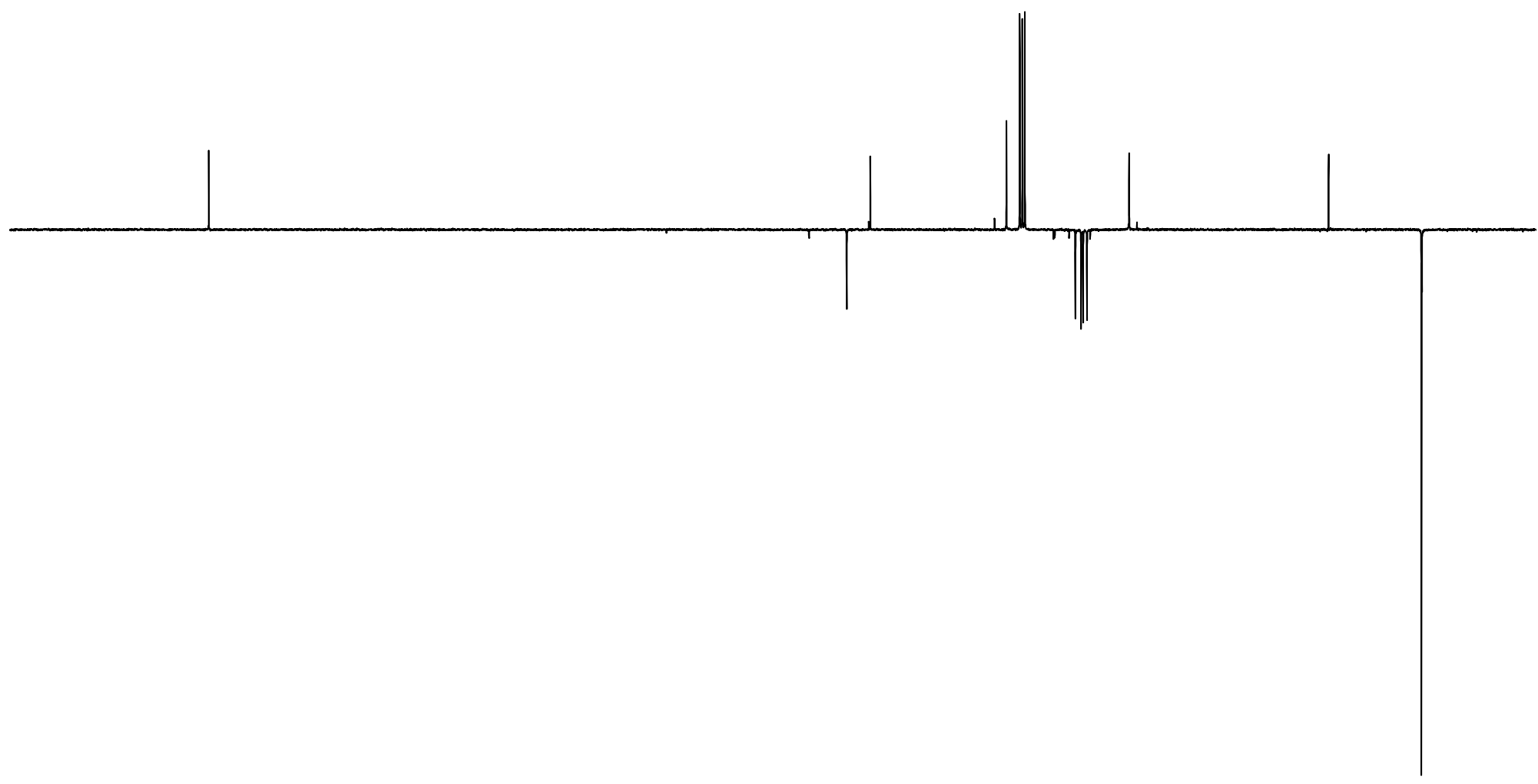


Compound 15, $400 \mathrm{MHz}, 295 \mathrm{~K}, \mathrm{CDCl}_{3}$

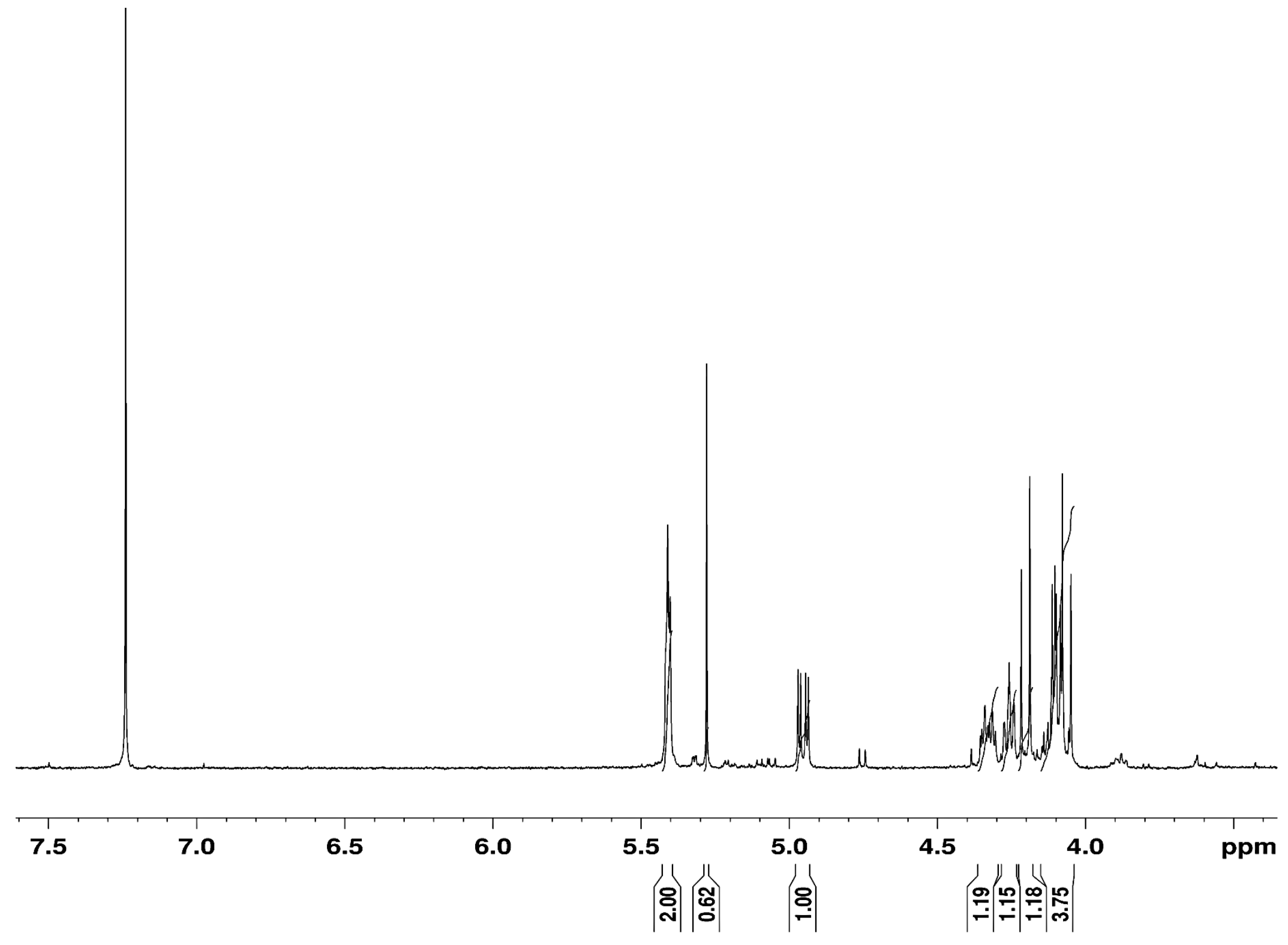


Compound 15, $101 \mathrm{MHz}, 297 \mathrm{~K}, \mathrm{CDCl}_{3}$
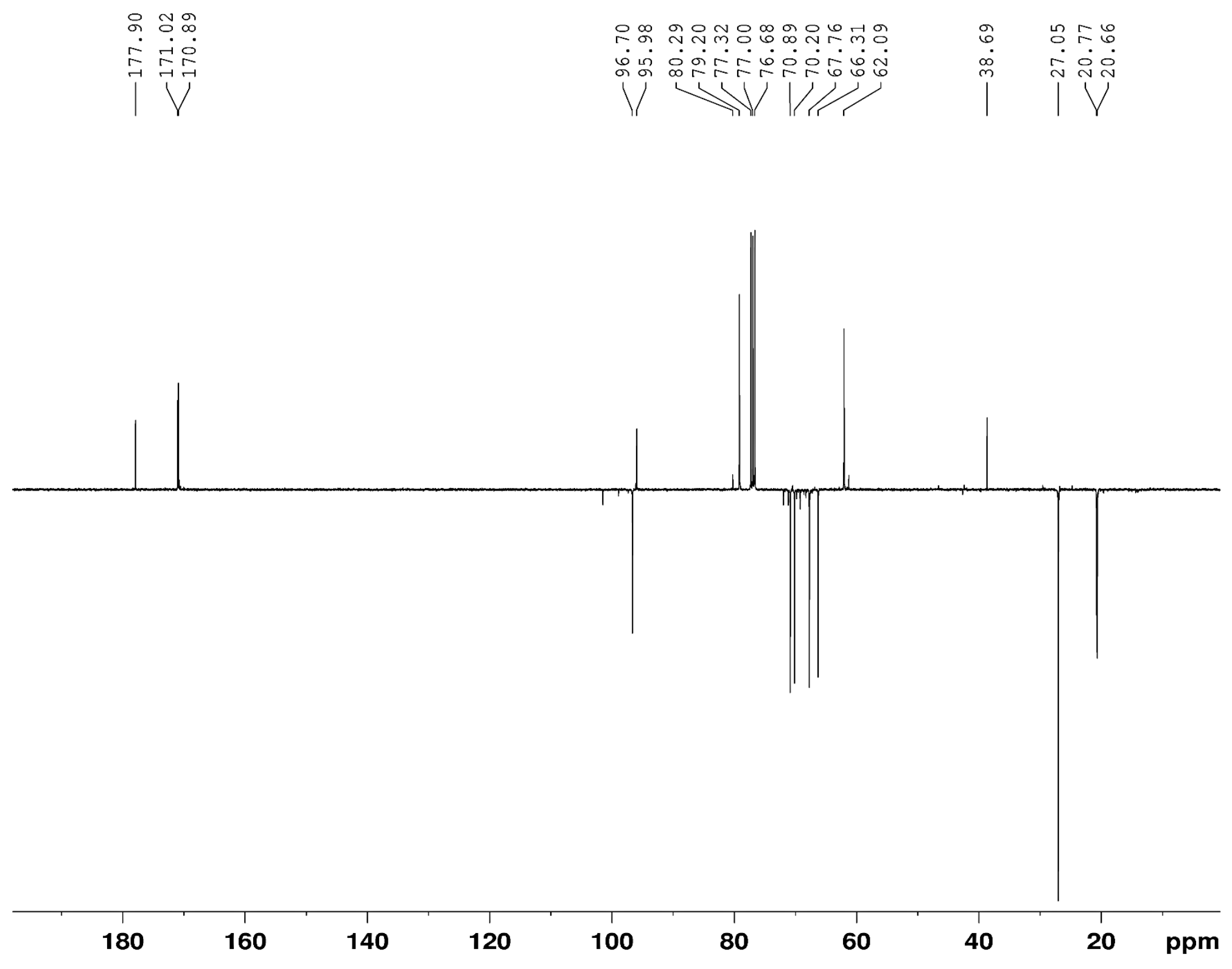
Compound 16, $600 \mathrm{MHz}, 296 \mathrm{~K}, \mathrm{CDCl}_{3}$

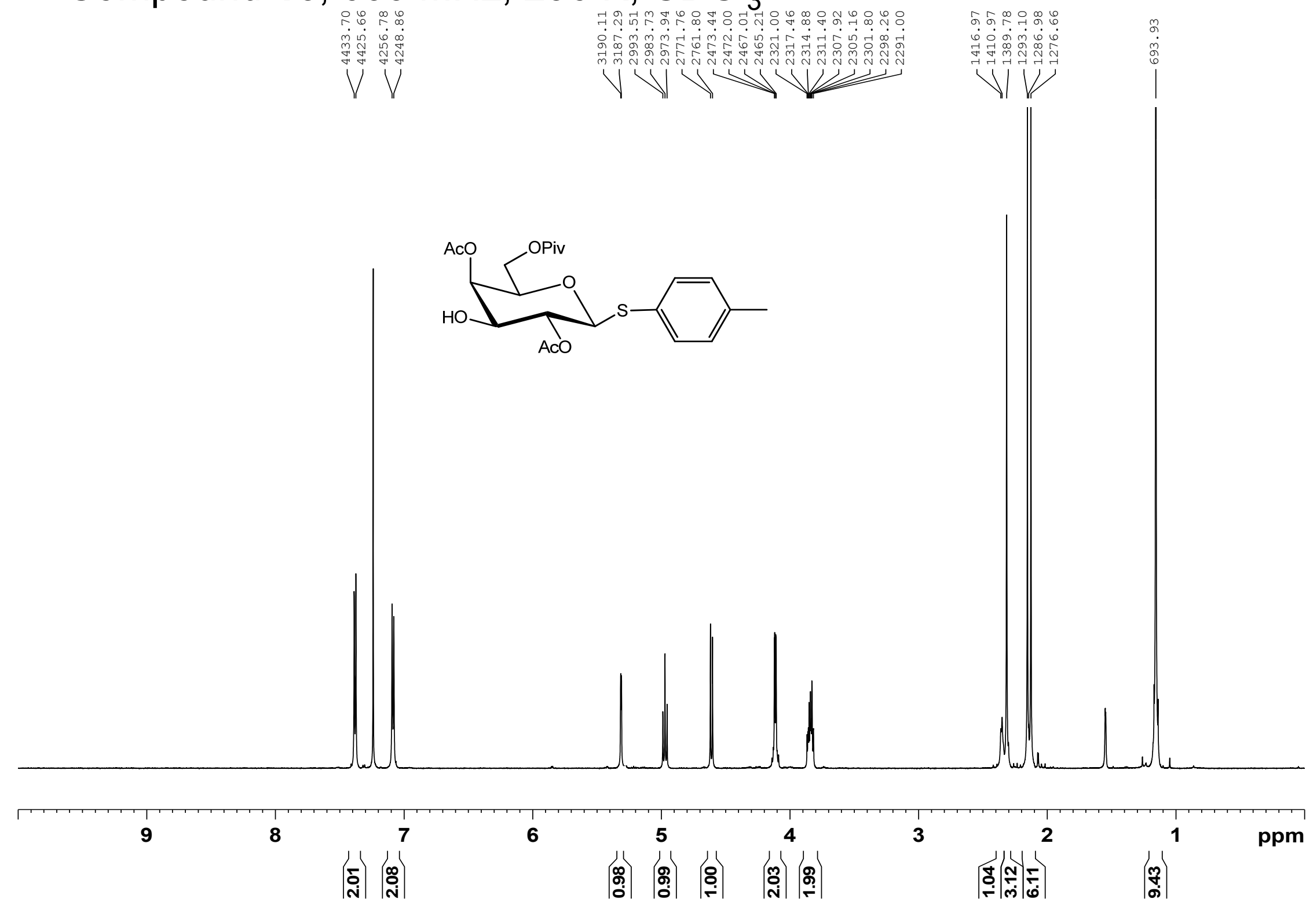


Compound 16, $151 \mathrm{MHz}, 296 \mathrm{~K}, \mathrm{CDCl}_{3}$

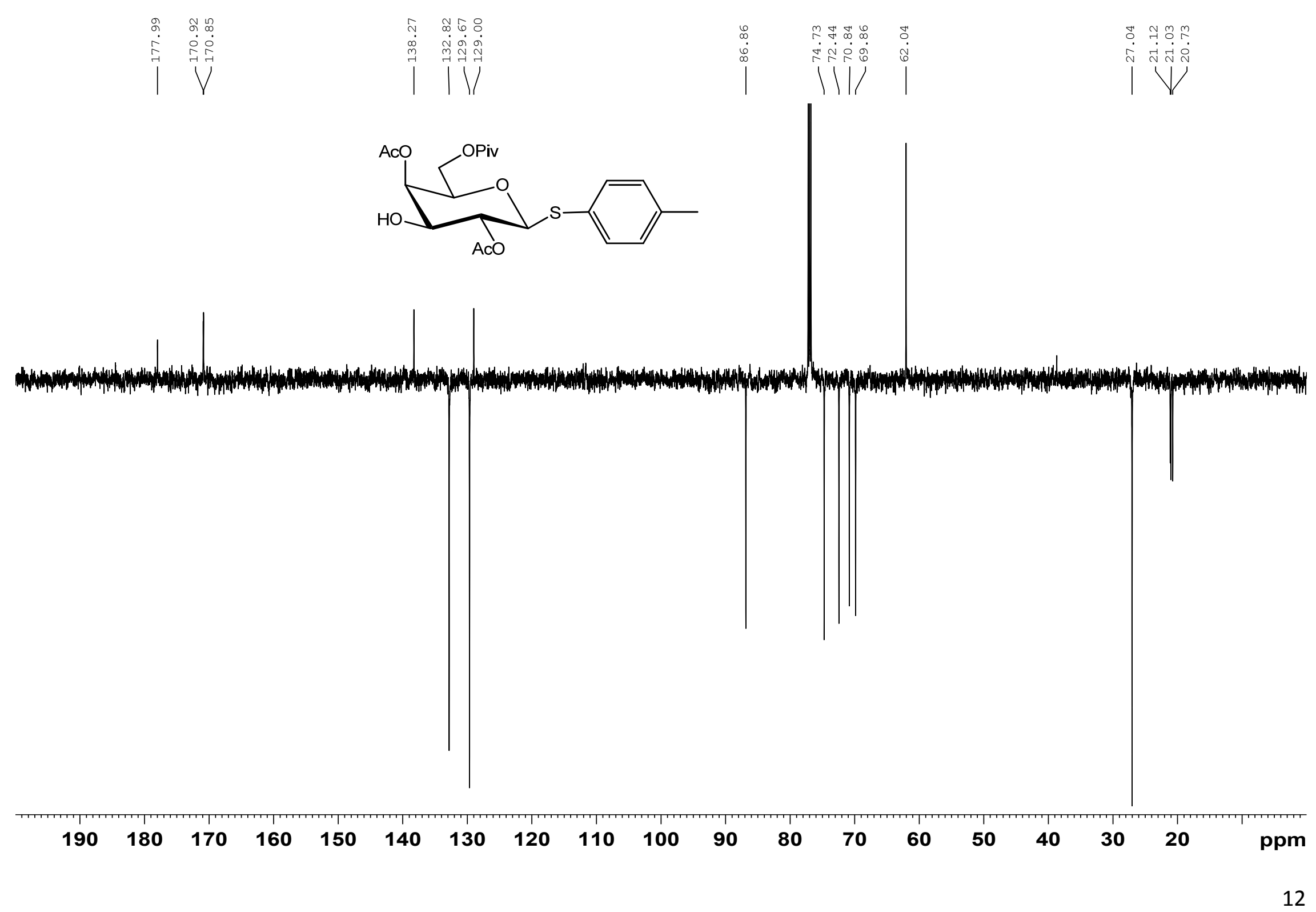


Compound 17, $600 \mathrm{MHz}, 296 \mathrm{~K}, \mathrm{CDCl}_{3}$

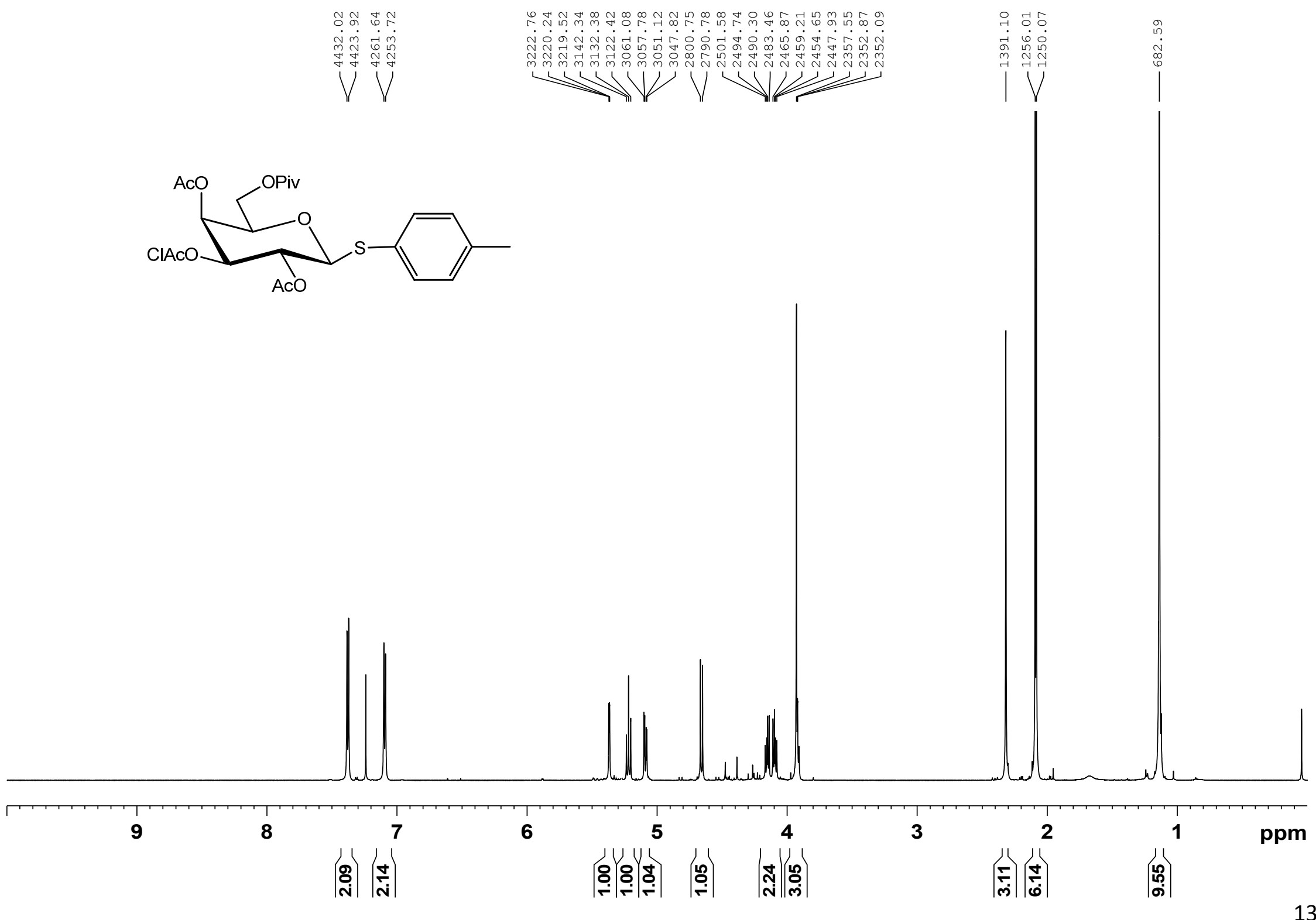


Compound 17, $151 \mathrm{MHz}, 296 \mathrm{~K}, \mathrm{CDCl}_{3}$

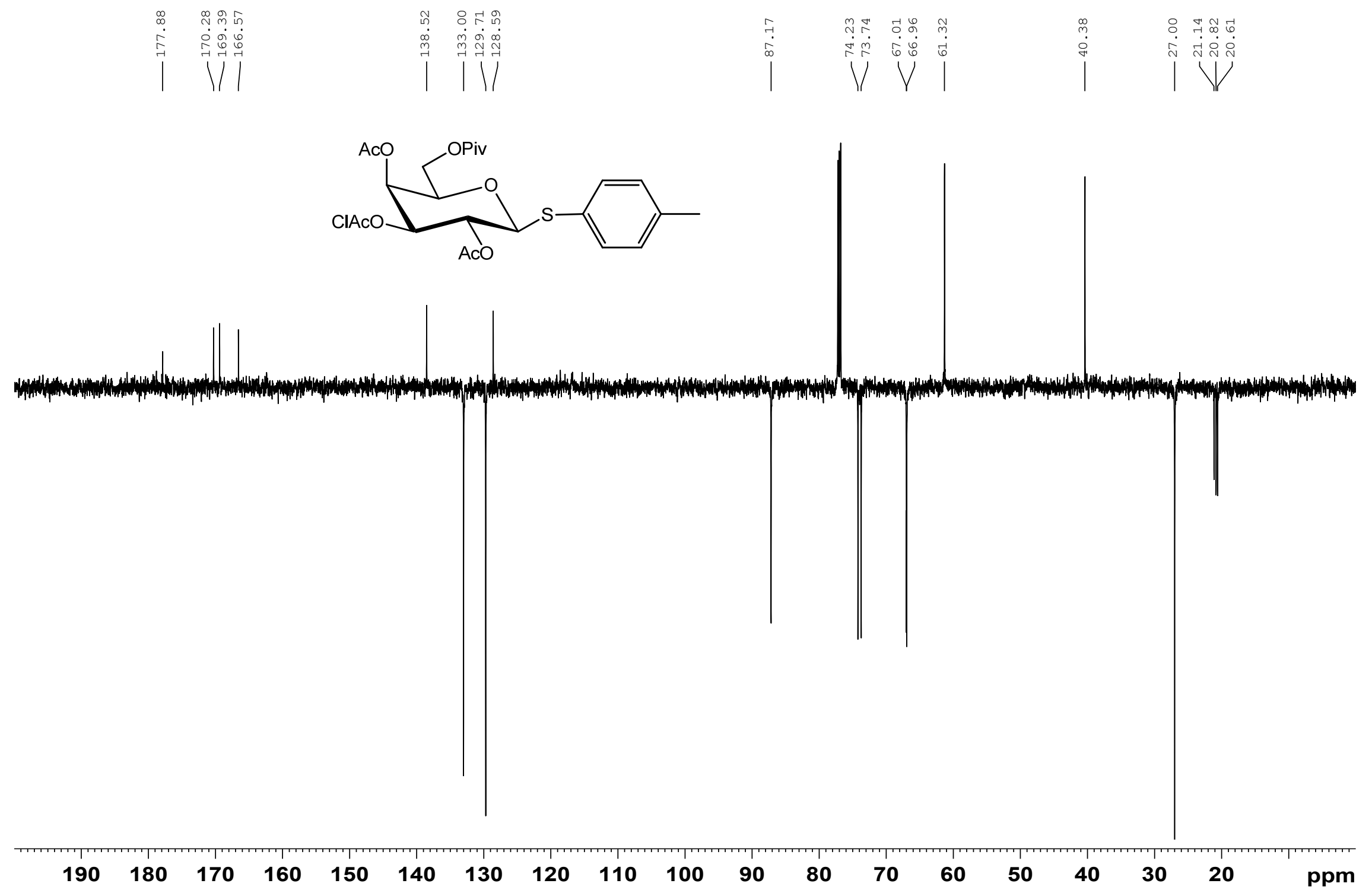


Compound 18, $400 \mathrm{MHz}, 296 \mathrm{~K}, \mathrm{CDCl}_{3}$

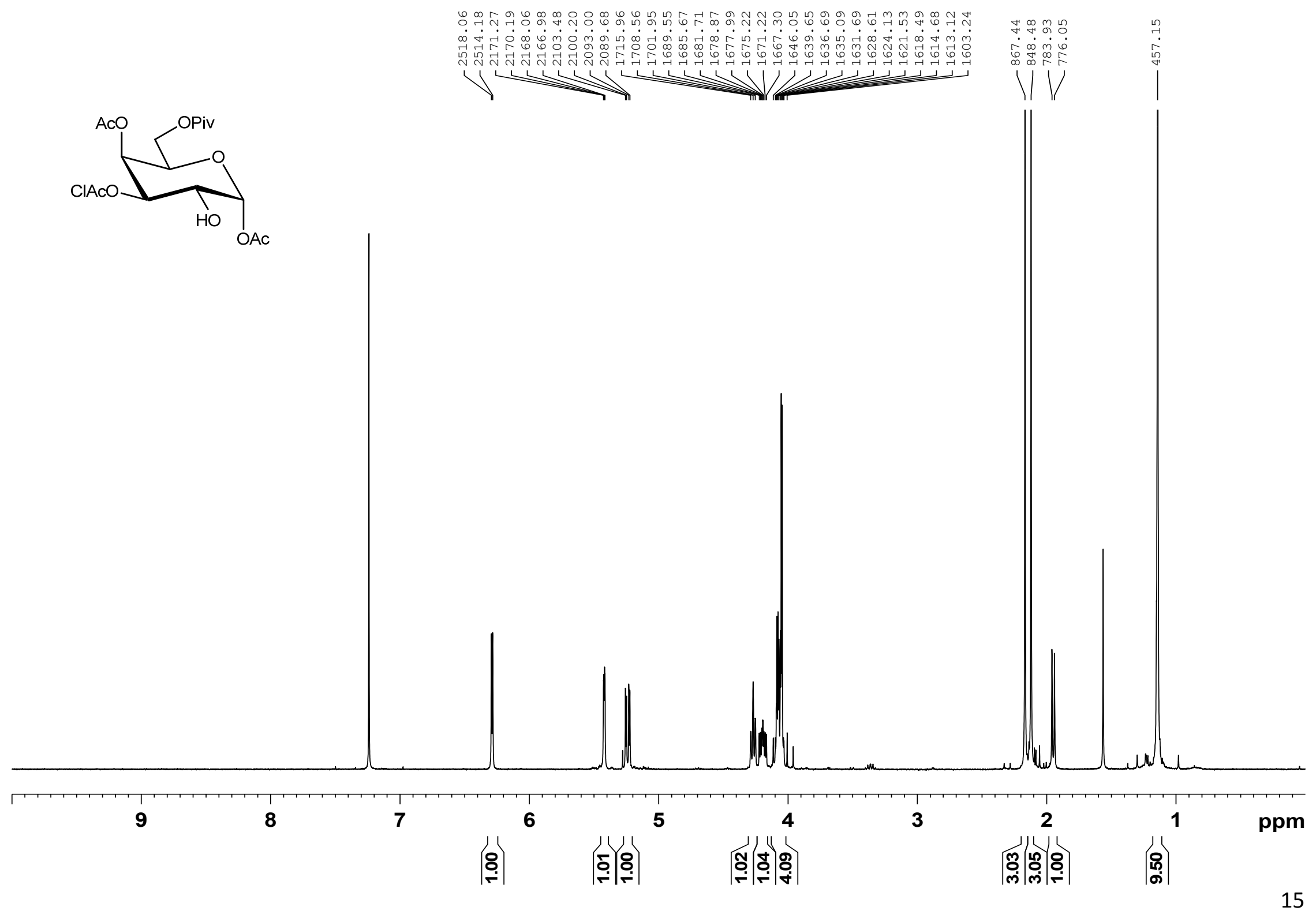


Compound 18, $101 \mathrm{MHz}, 296 \mathrm{~K}, \mathrm{CDCl}_{3}$

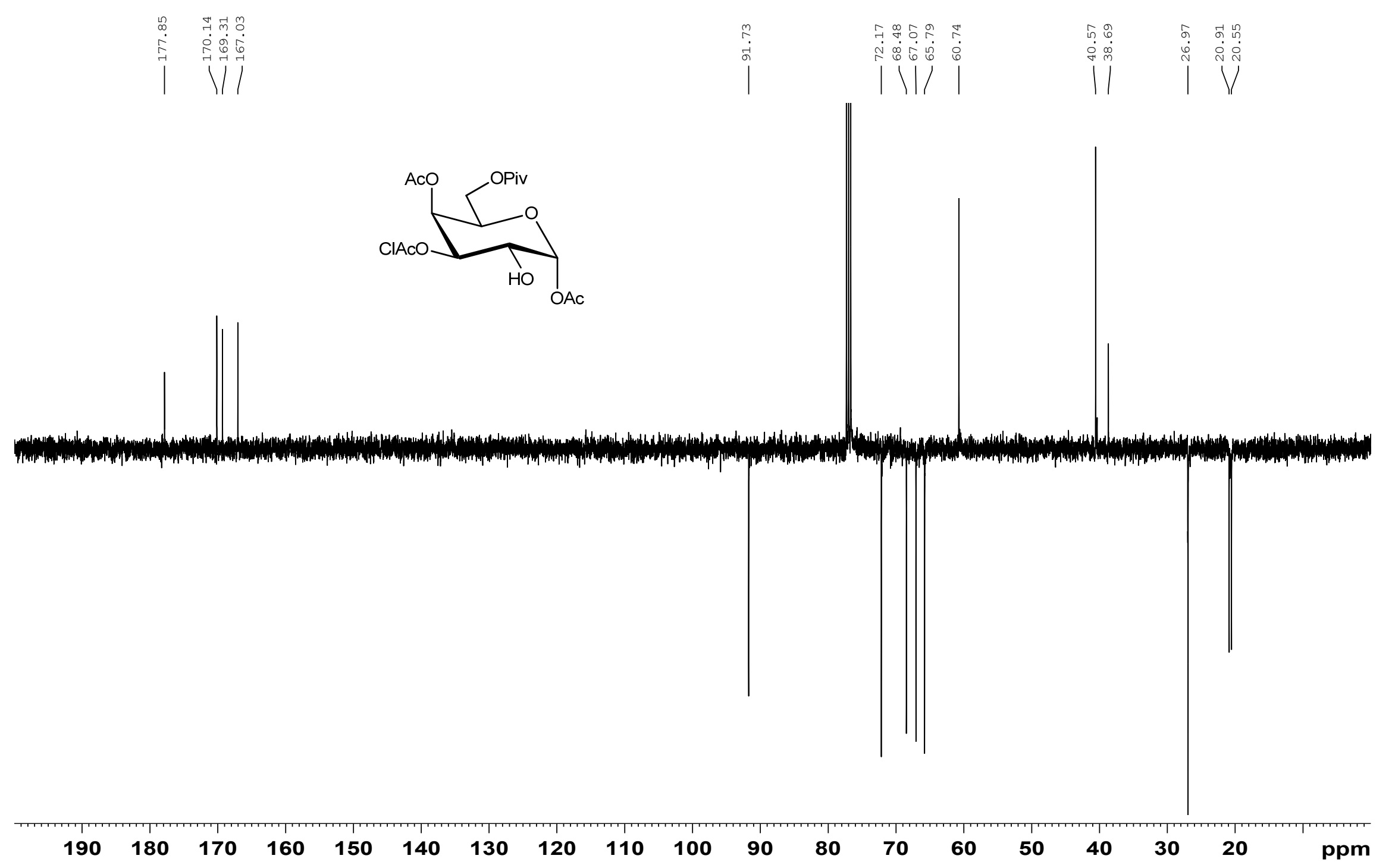


Compound 6, $400 \mathrm{MHz}, 296 \mathrm{~K}, \mathrm{CDCl}_{3}$

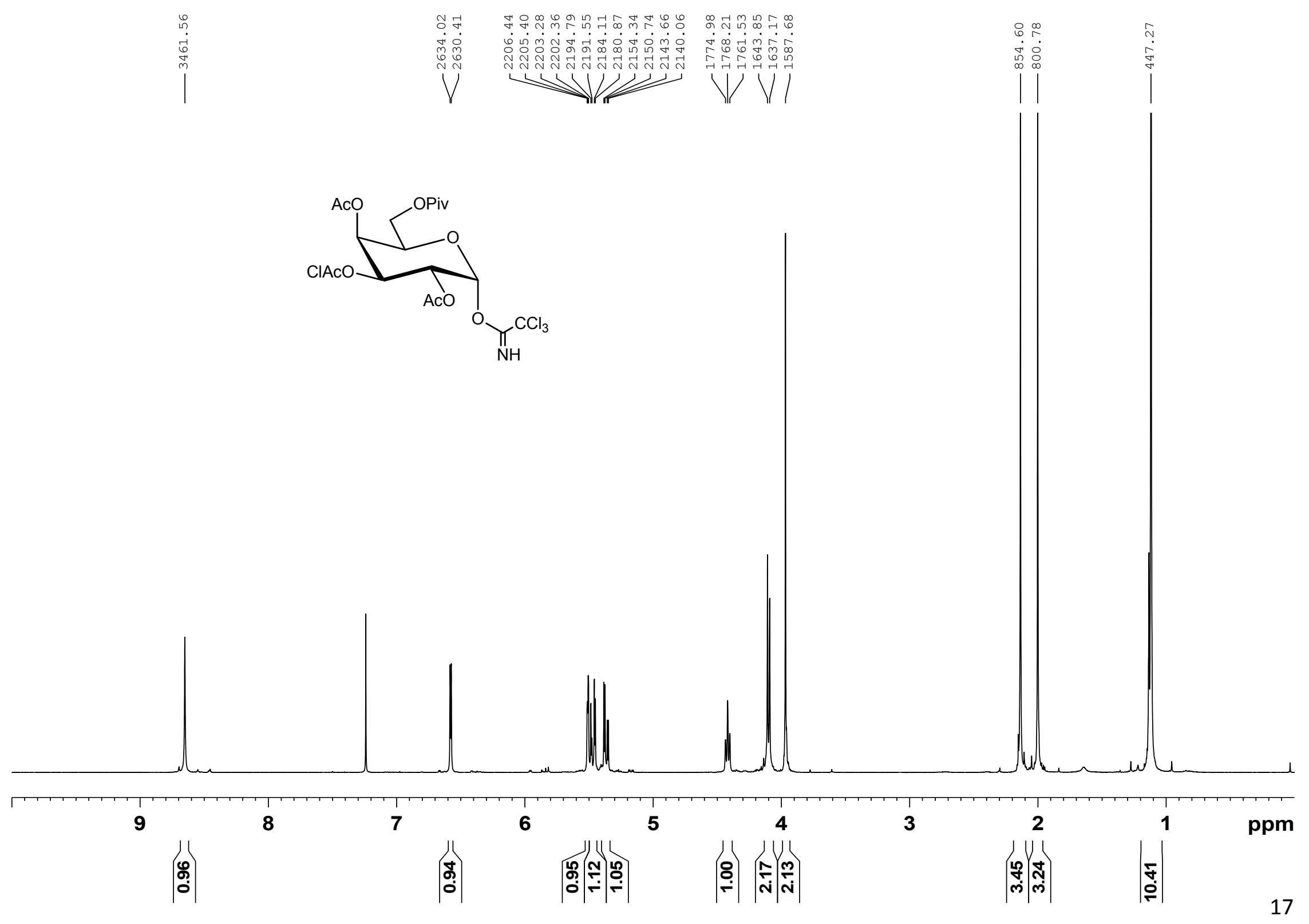


Compound 6, $101 \mathrm{MHz}, 296 \mathrm{~K}, \mathrm{CDCl}_{3}$

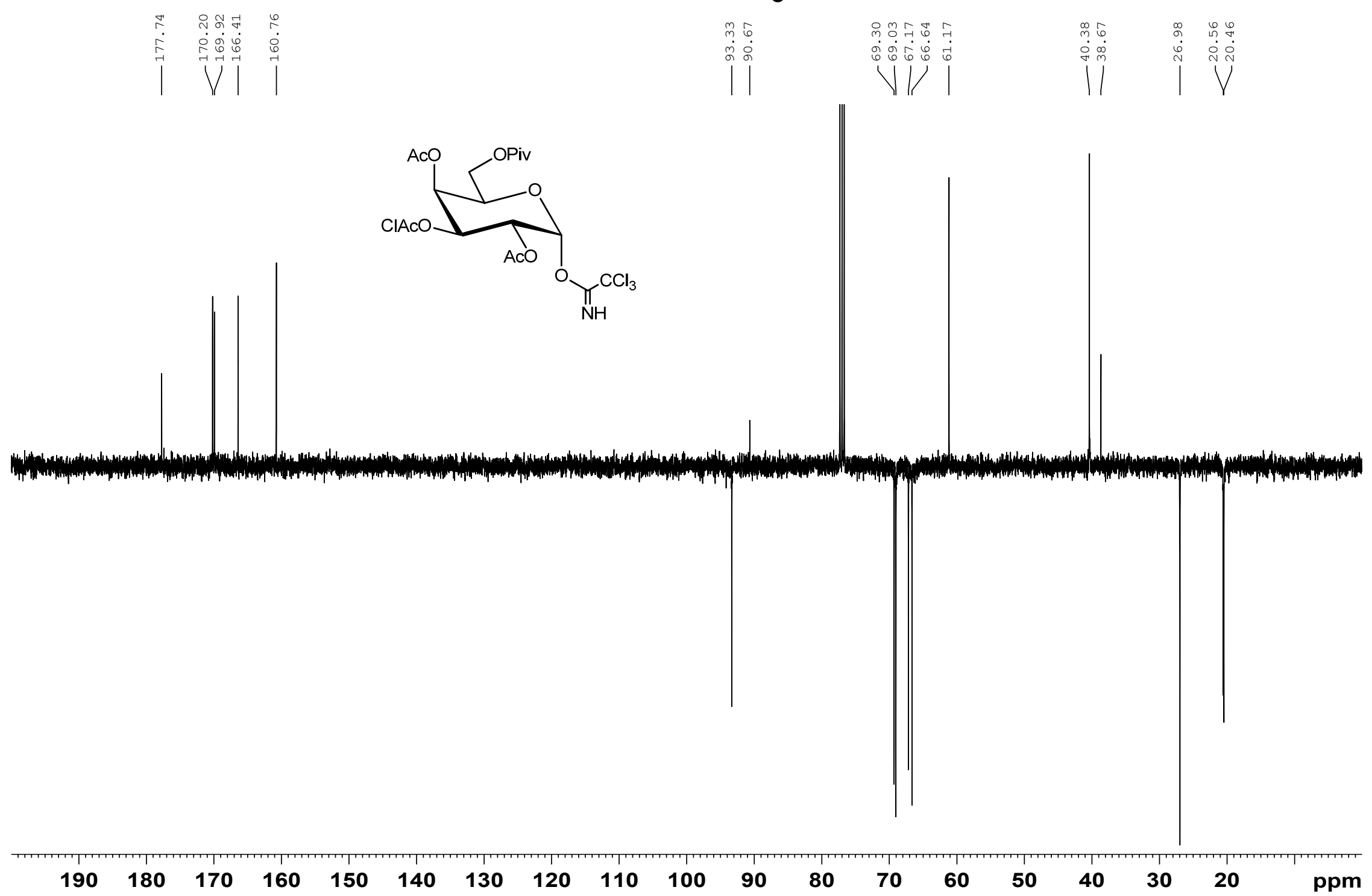




\section{Compound 19, $400 \mathrm{MHz}, 296 \mathrm{~K}, \mathrm{CDCl}_{3}$}

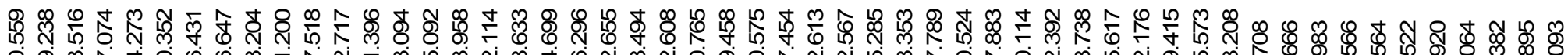

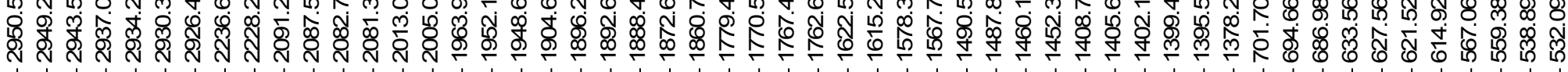

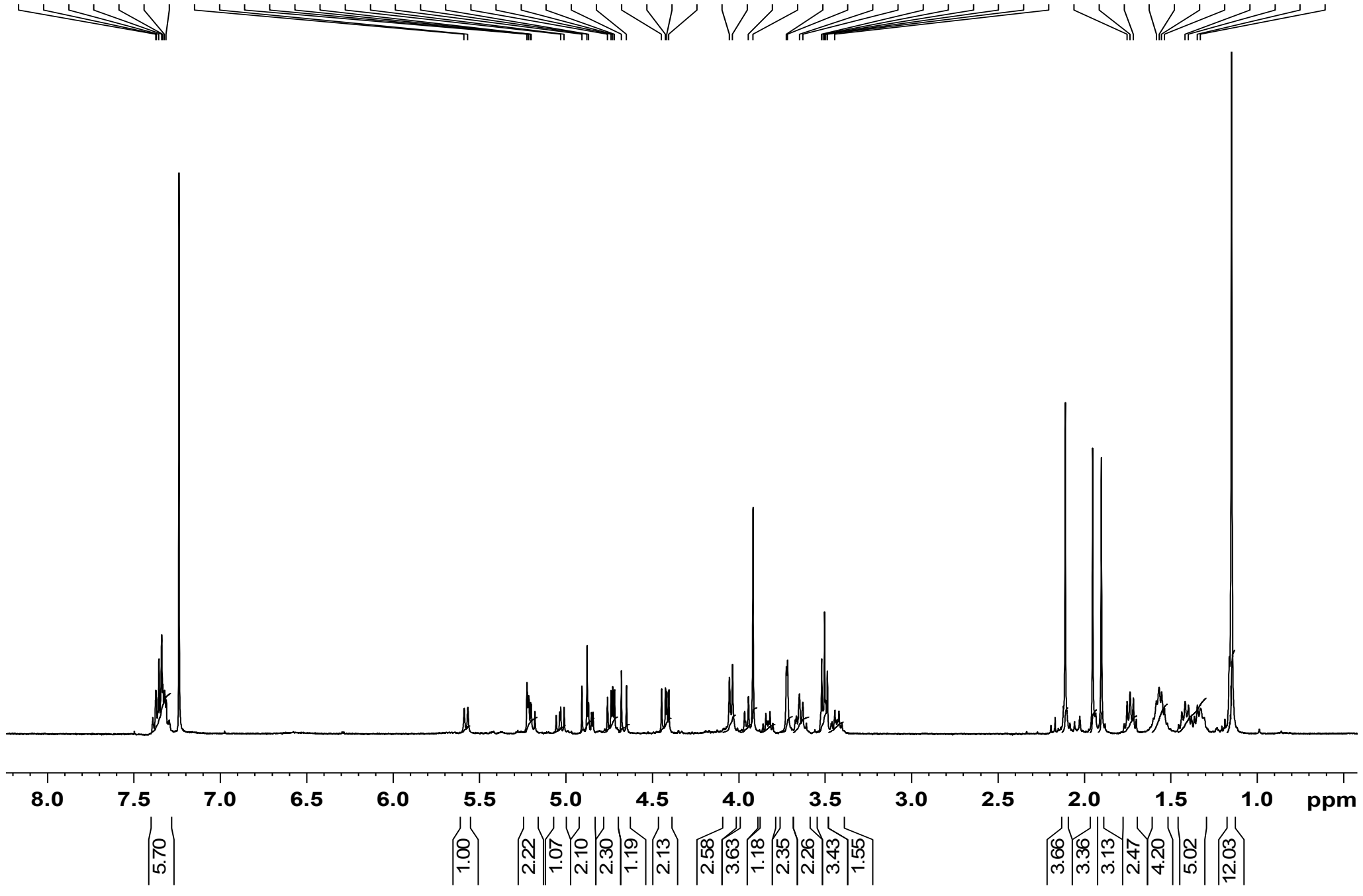


Compound 19, $101 \mathrm{MHz}, 296 \mathrm{~K}, \mathrm{CDCl}_{3}$

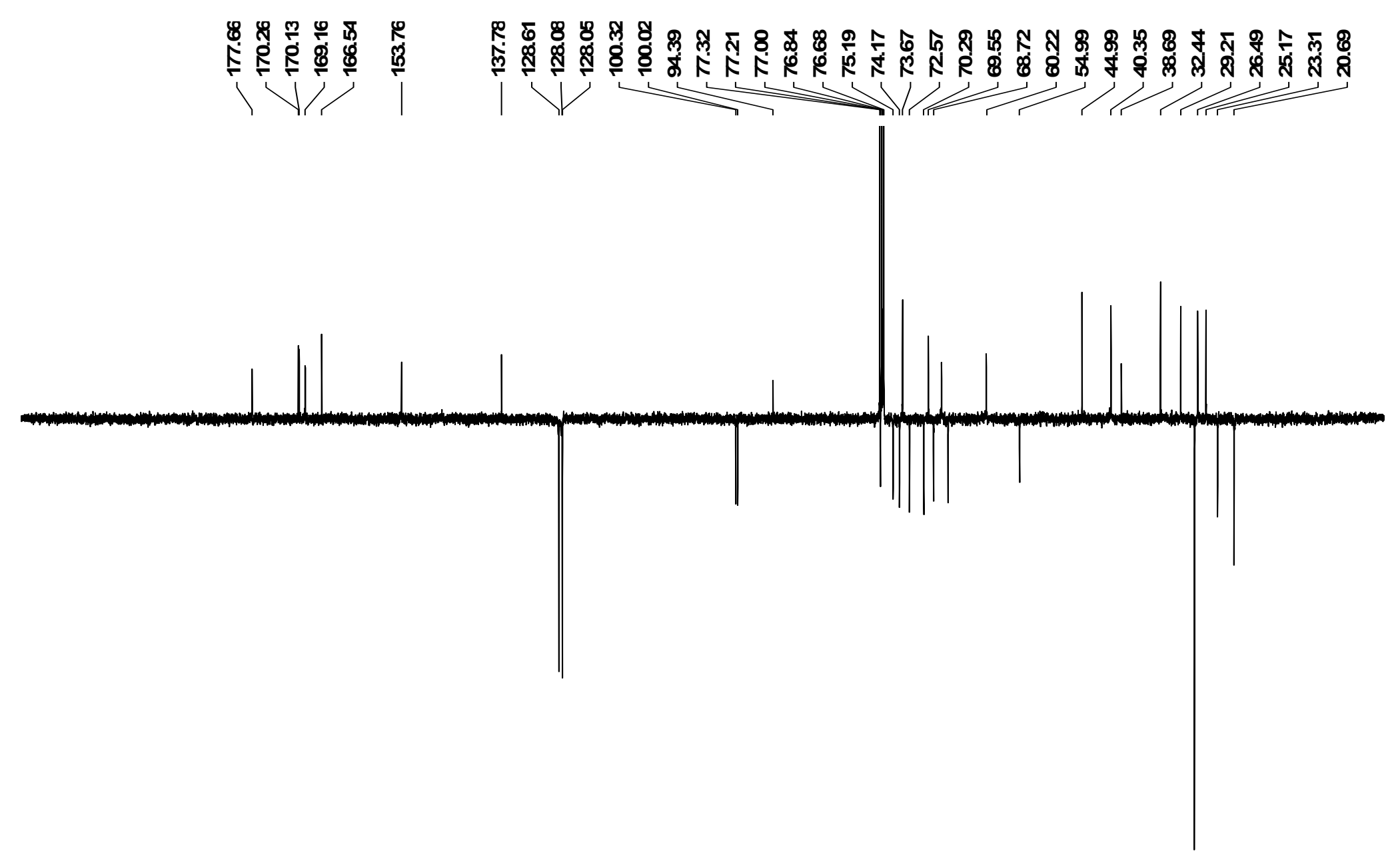




\section{Compound 20, $400 \mathrm{MHz}, 296 \mathrm{~K}, \mathrm{CDCl}_{3}$}

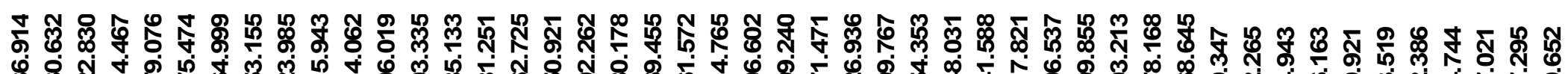

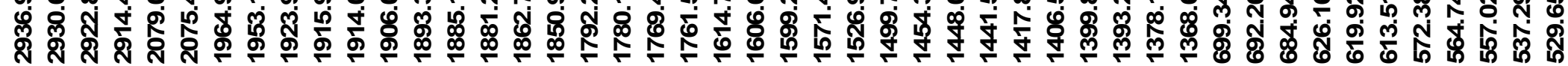

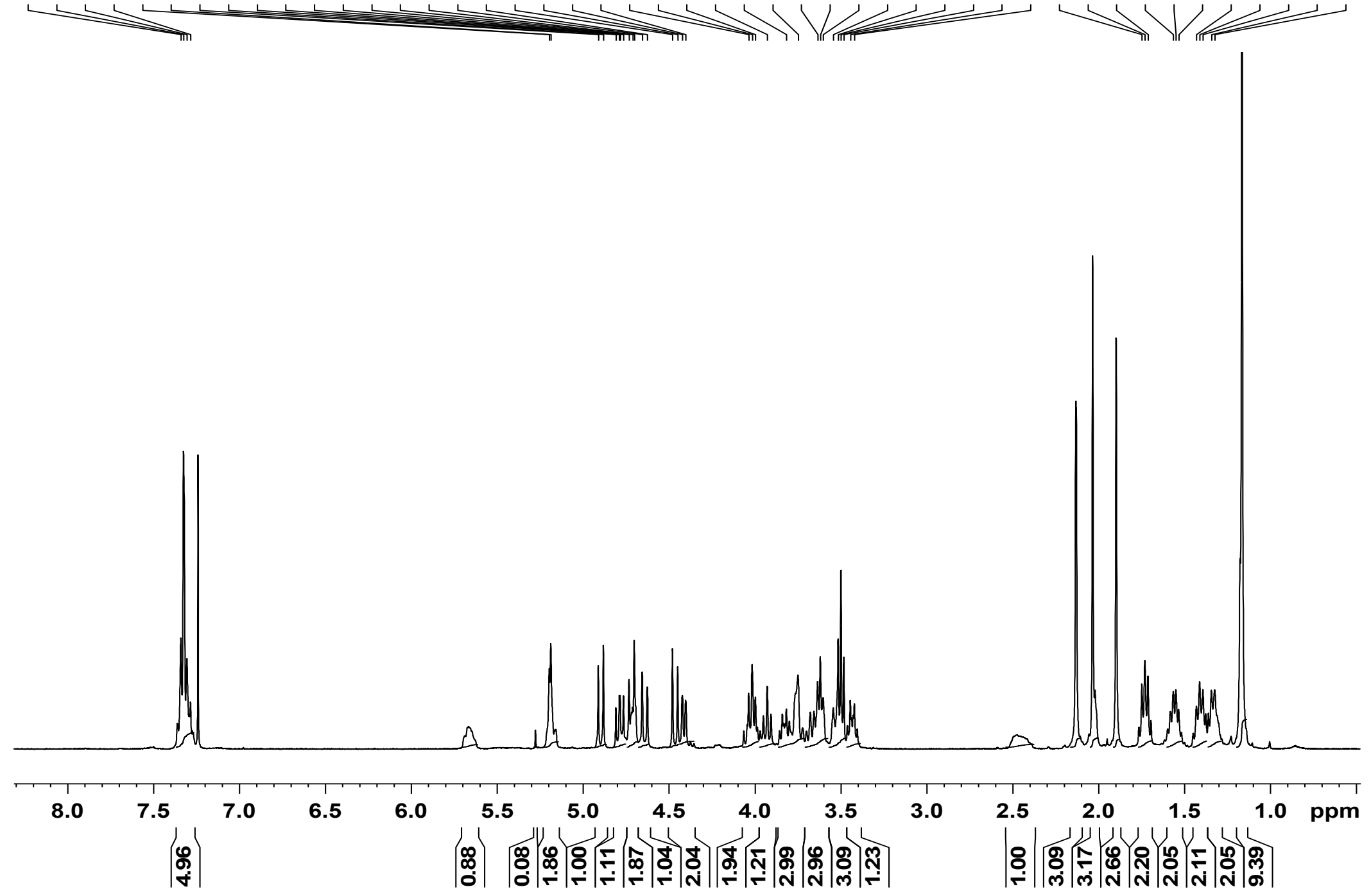


Compound 20, $101 \mathrm{MHz}, 297 \mathrm{~K}, \mathrm{CDCl}_{3}$

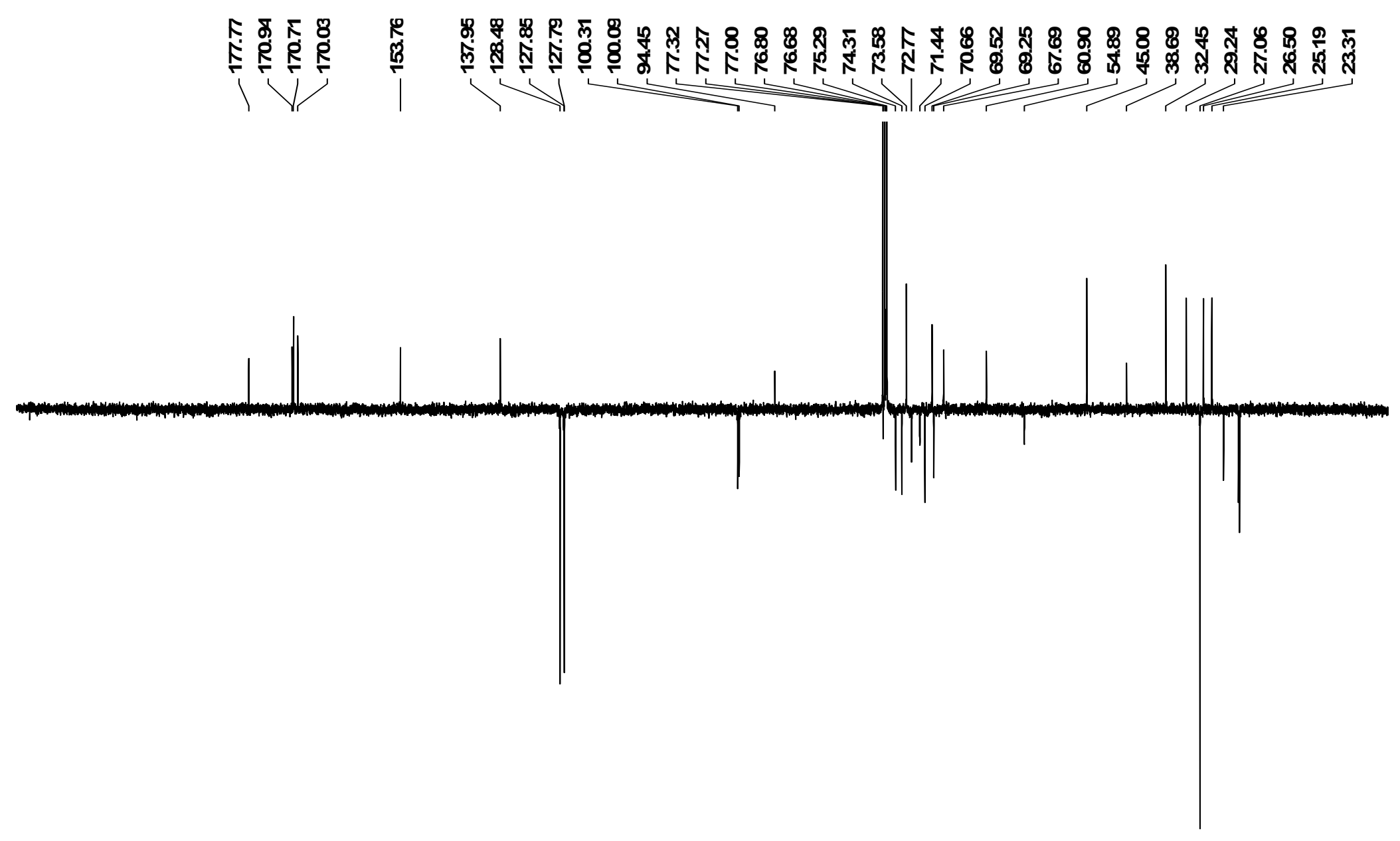

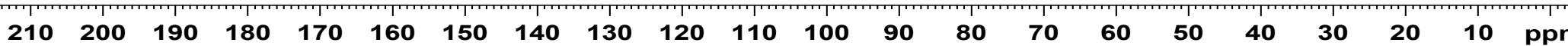




\section{Compound 21, $400 \mathrm{MHz}, 296 \mathrm{~K}, \mathrm{CDCl}_{3}$}

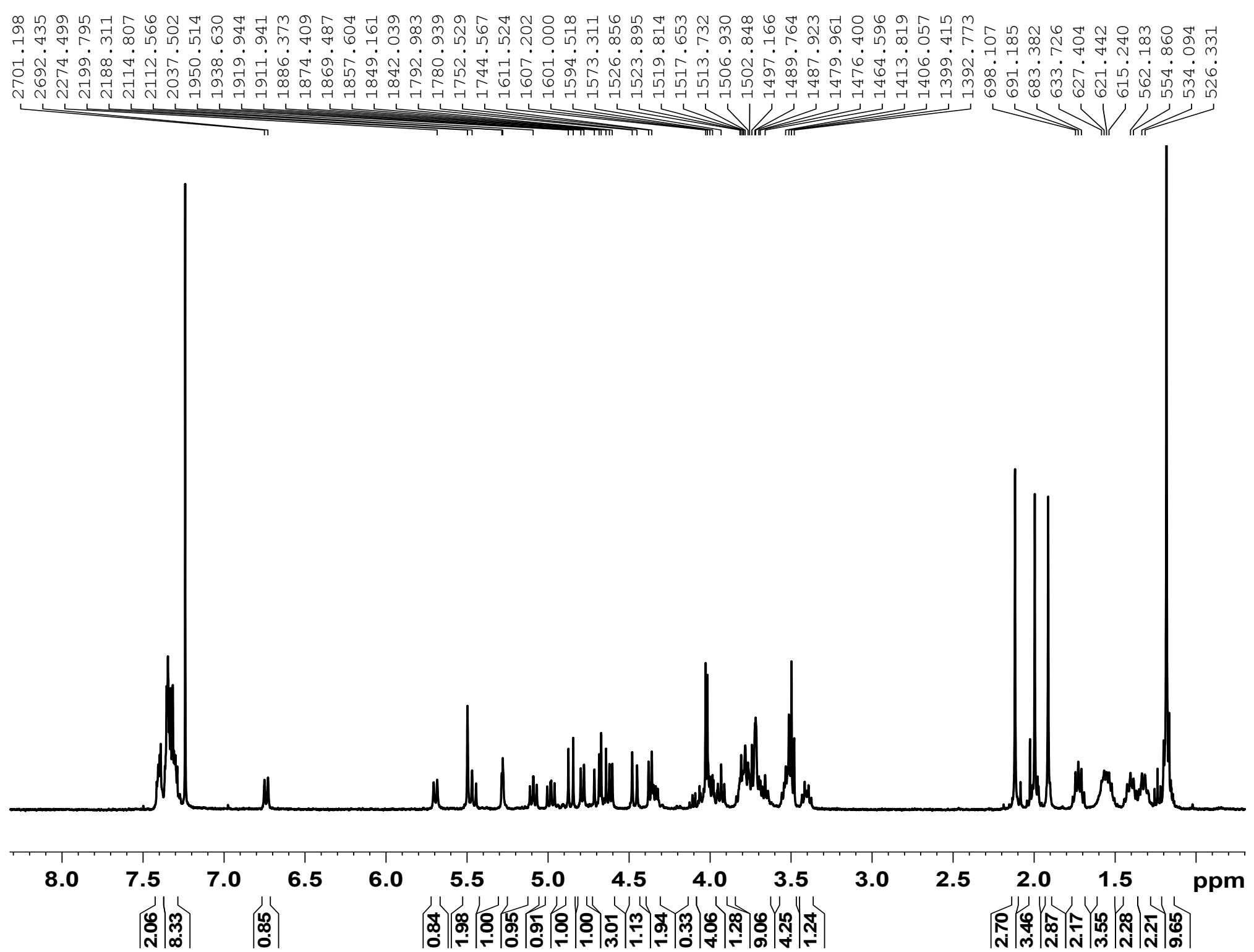


Compound 21, $101 \mathrm{MHz}, 296 \mathrm{~K}, \mathrm{CDCl}_{3}$

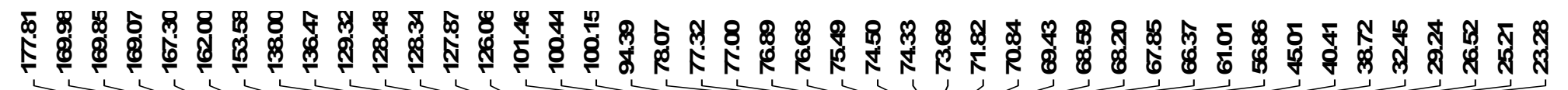

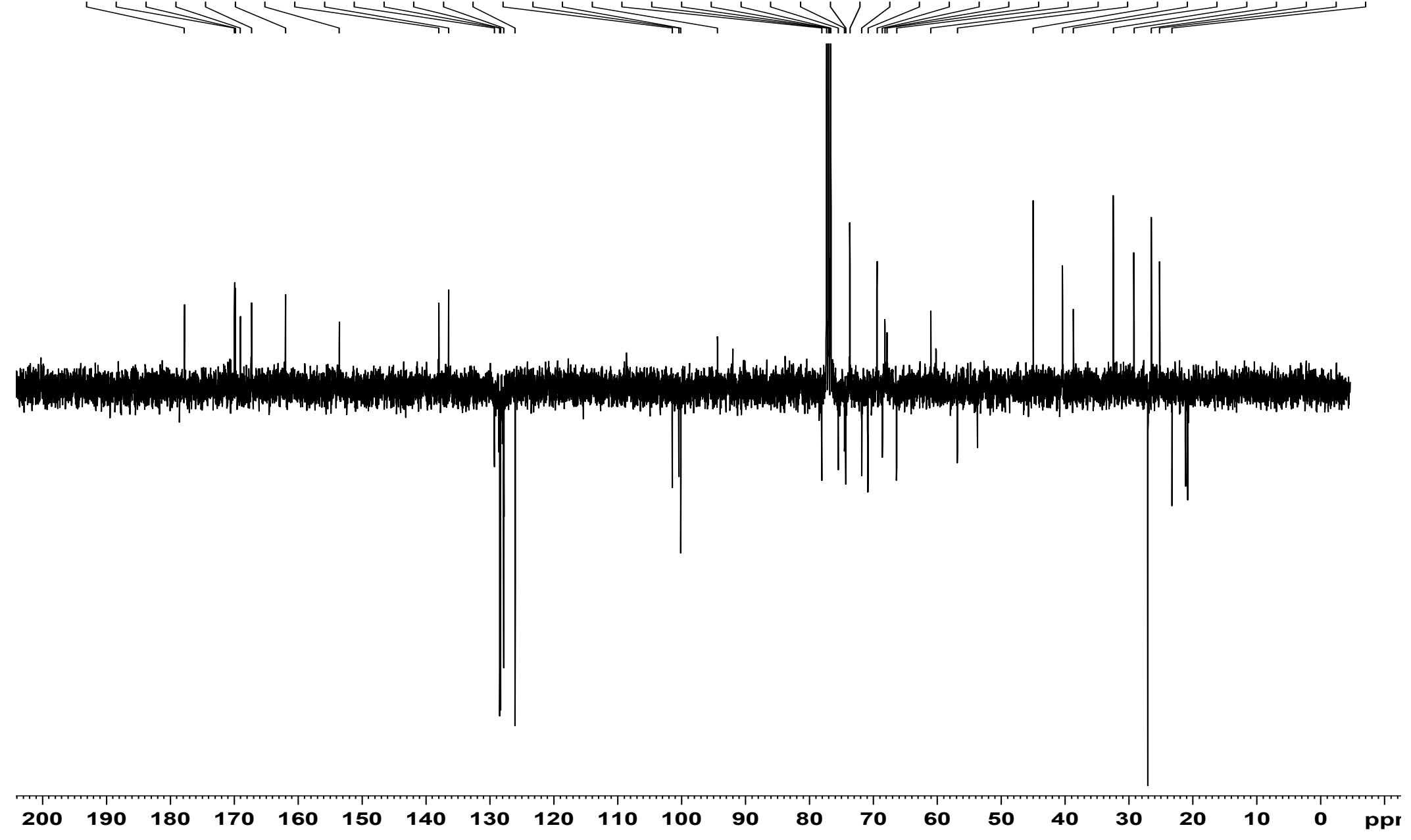


Compound 22, $400 \mathrm{MHz}, 295 \mathrm{~K}, \mathrm{CDCl}_{3}$

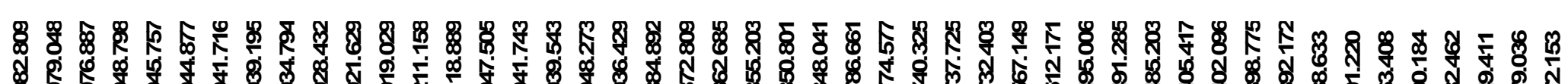
M
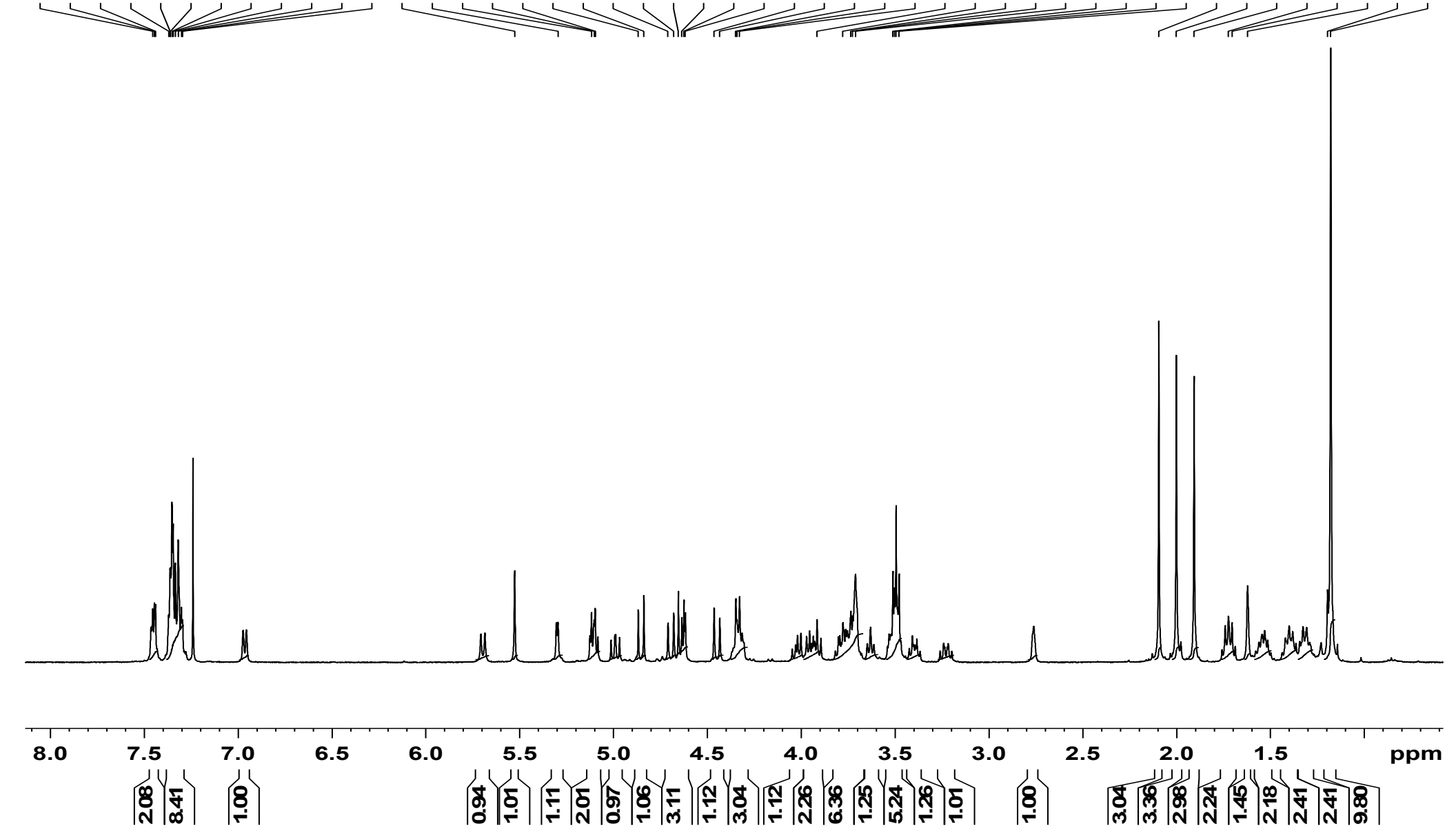
Compound 22, $101 \mathrm{MHz}, 297 \mathrm{~K}, \mathrm{CDCl}_{3}$

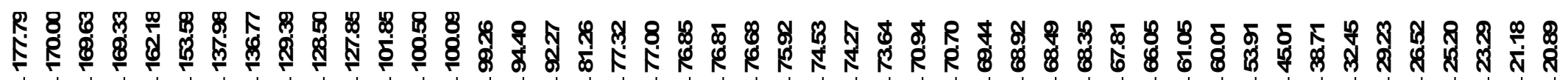

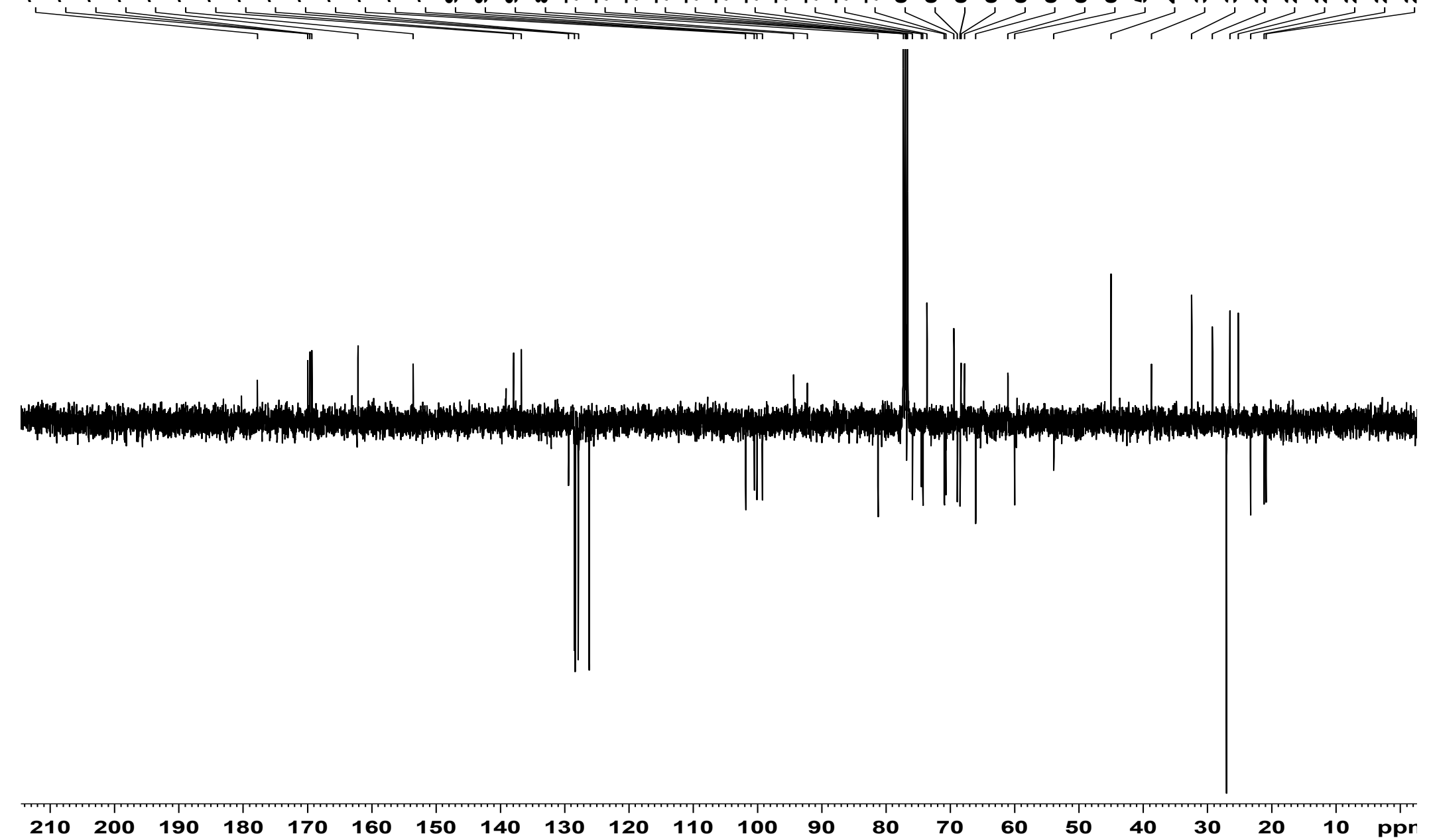


Compound 23, $600 \mathrm{MHz}, 295 \mathrm{~K}, \mathrm{CDCl}_{3}$

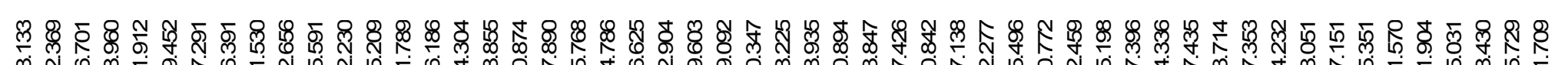

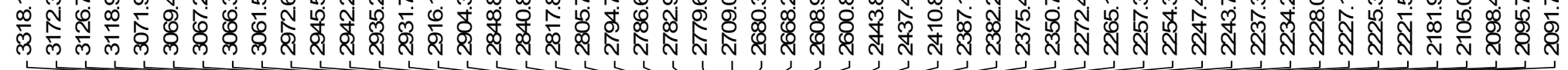
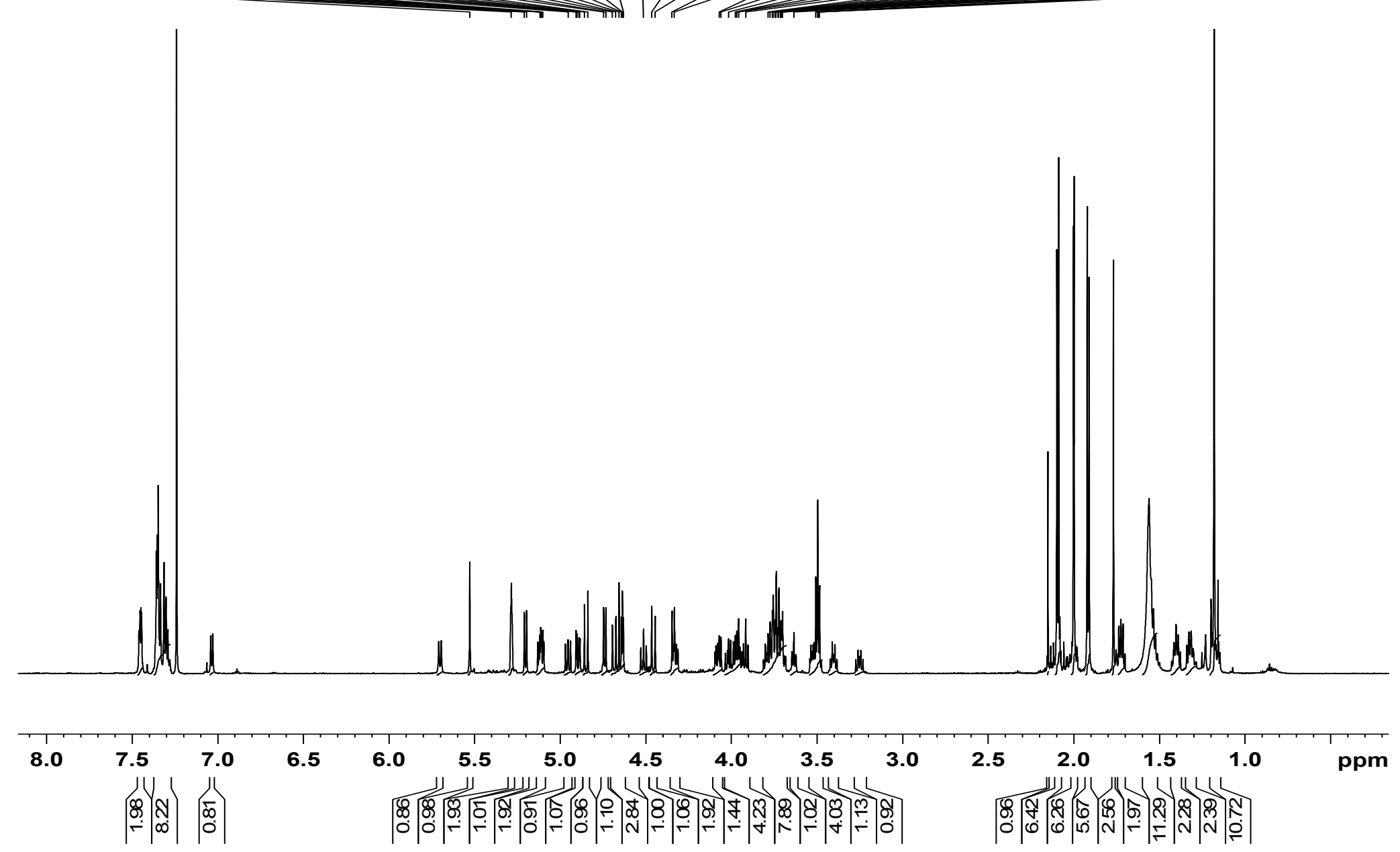
Compound 23, $151 \mathrm{MHz}, 295 \mathrm{~K}, \mathrm{CDCl}_{3}$

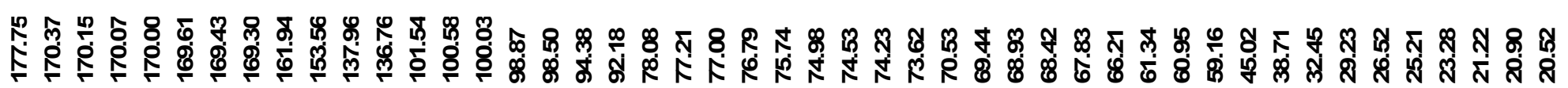

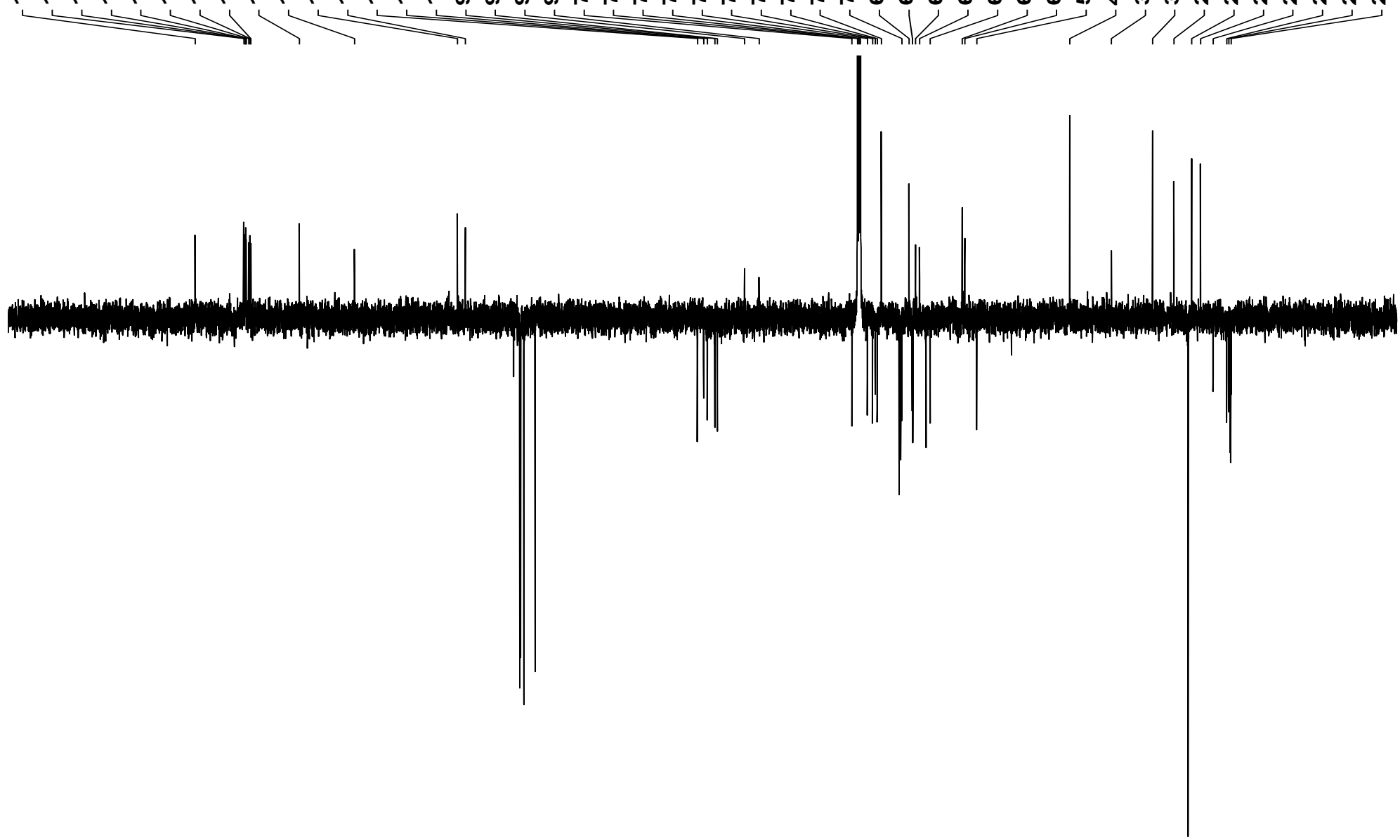

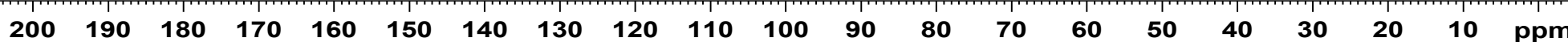


Compound 24, $400 \mathrm{MHz}, 296 \mathrm{~K}, \mathrm{CD}_{3} \mathrm{OD}$

mon

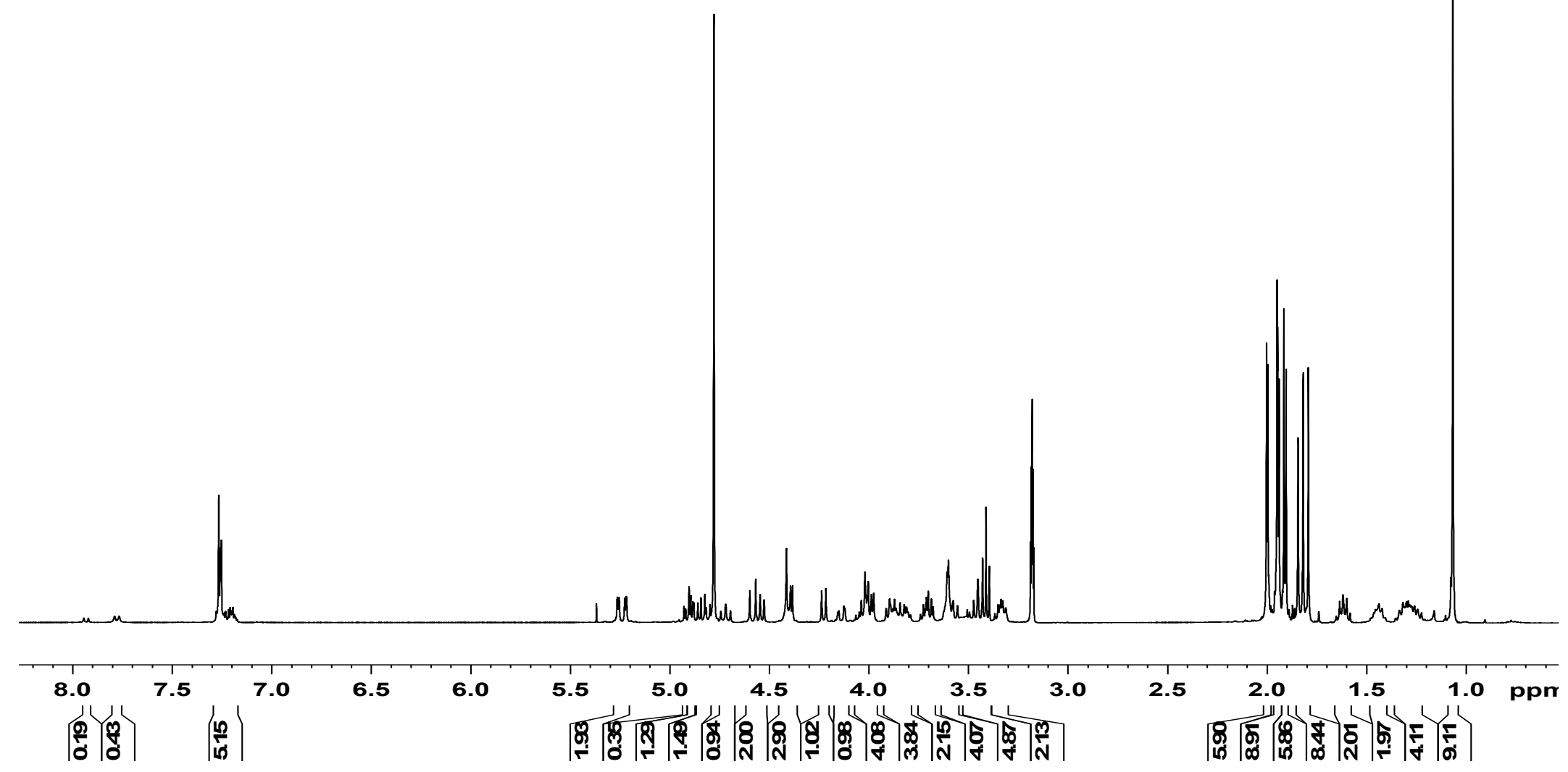


Compound 24, $101 \mathrm{MHz}, 296 \mathrm{~K}, \mathrm{CD}_{3} \mathrm{OD}$

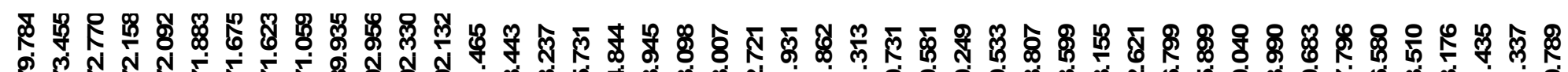
Q

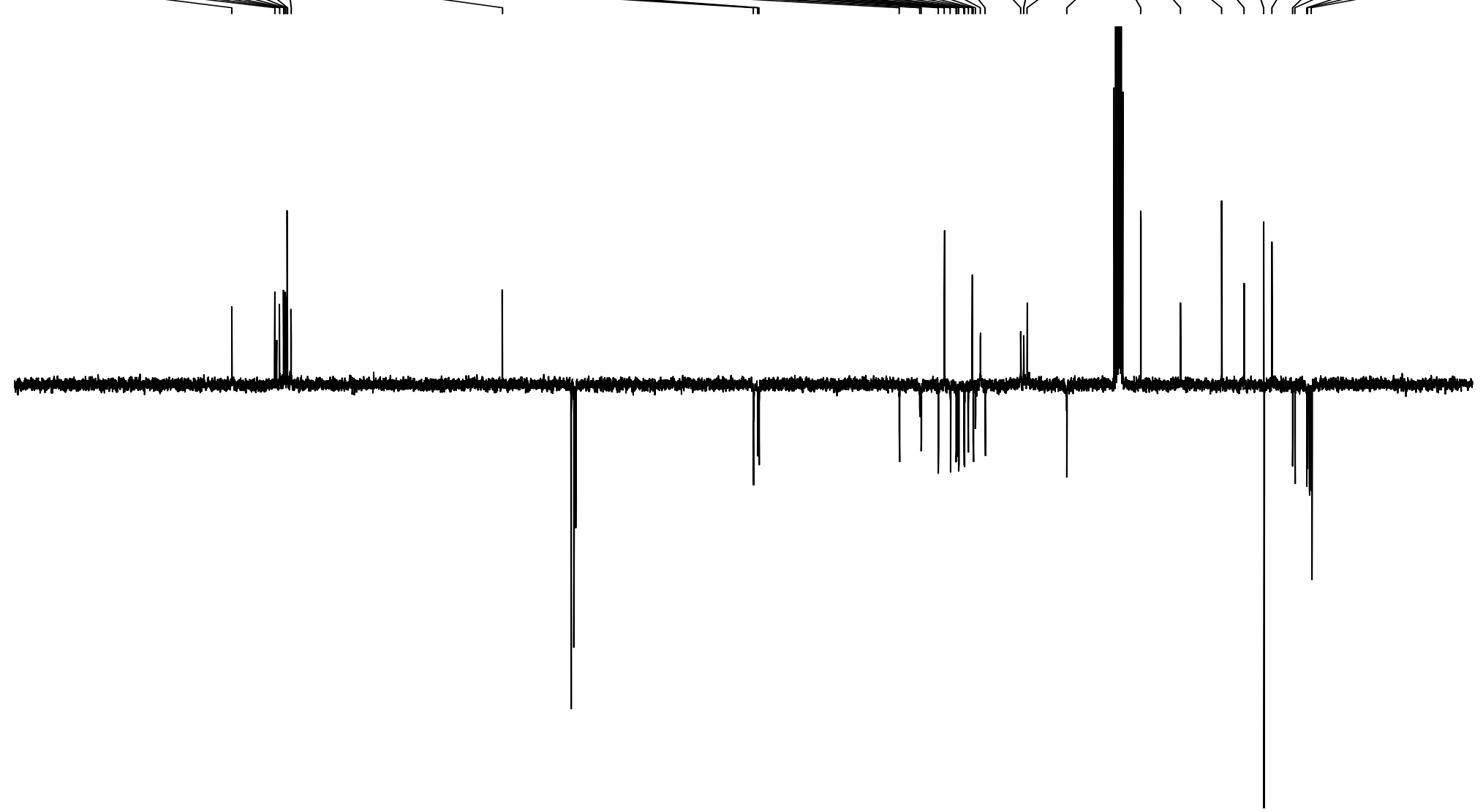

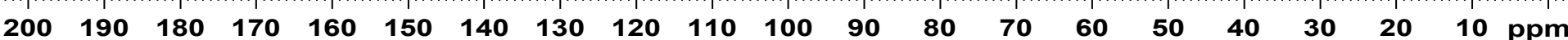


Compound 25, $600 \mathrm{MHz}, 297 \mathrm{~K}, \mathrm{CDCl}_{3}$
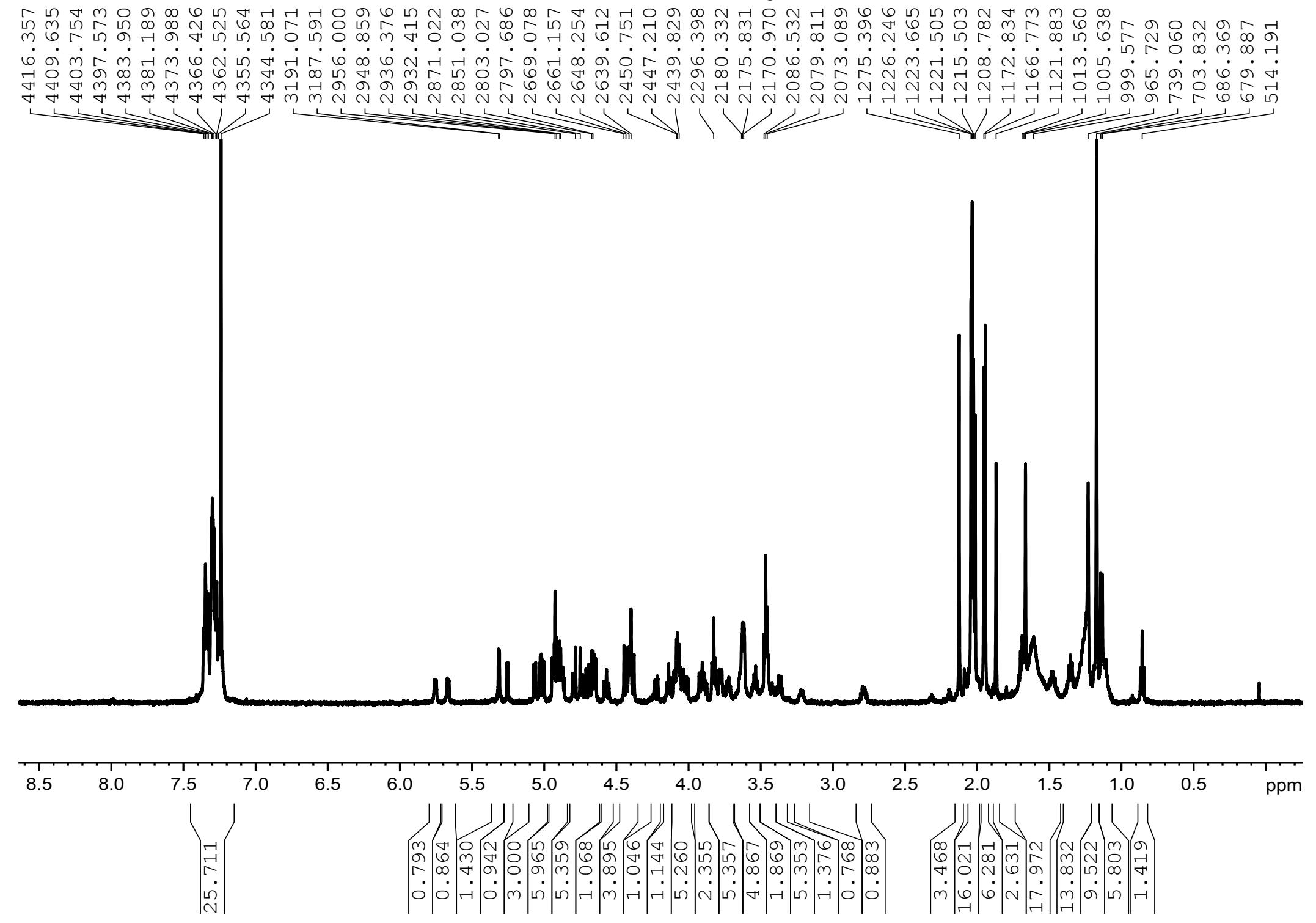


\section{Compound 25, $151 \mathrm{MHz}, 297 \mathrm{~K}, \mathrm{CDCl}_{3}$}

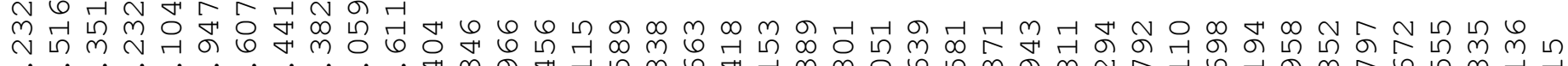

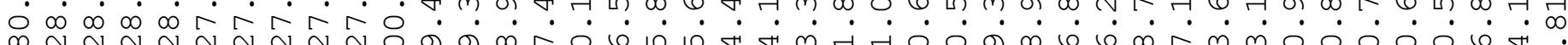

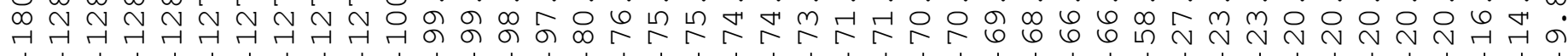

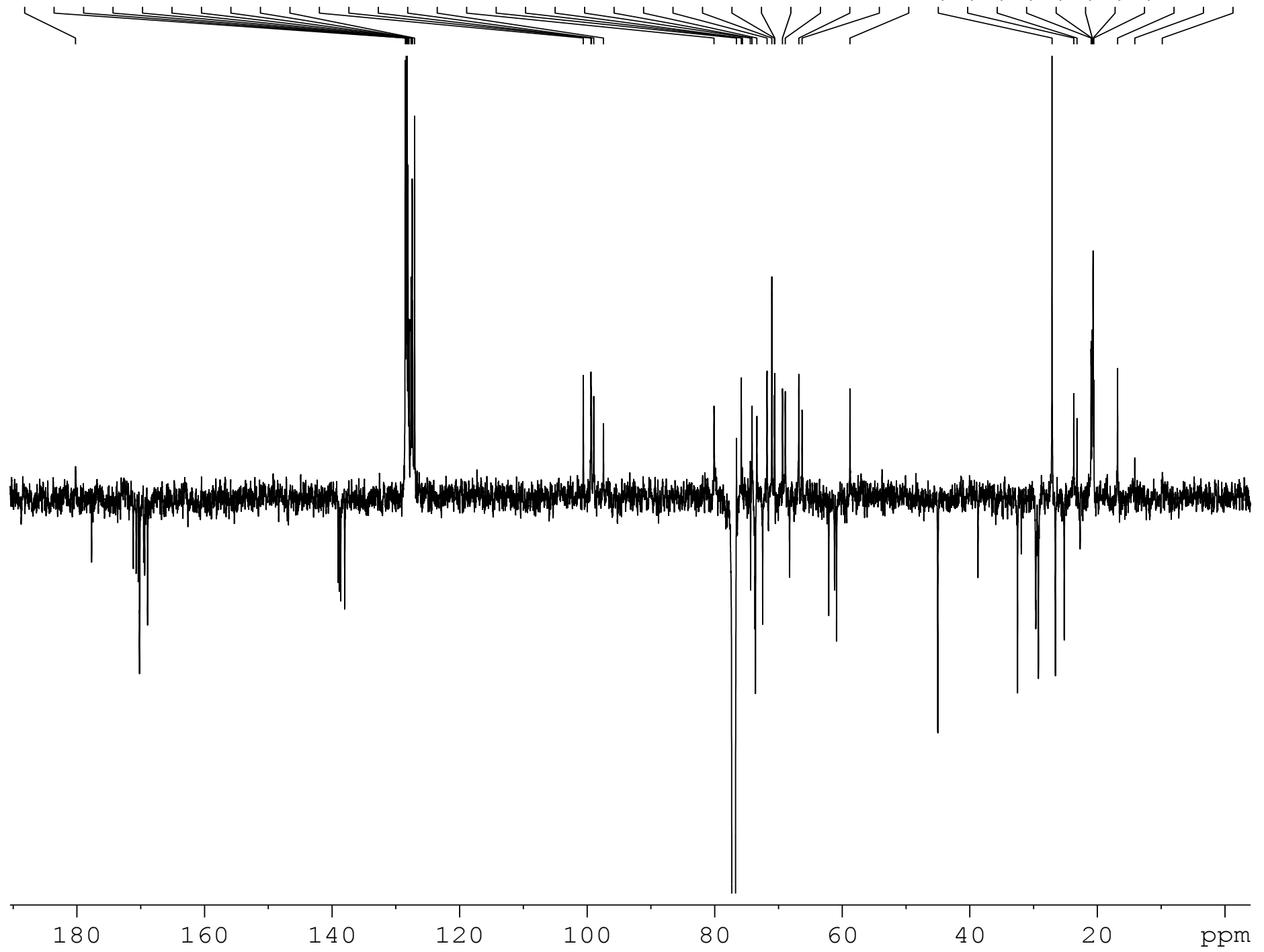


Compound 26, $400 \mathrm{MHz}, 296 \mathrm{~K}, \mathrm{CDCl}_{3}$

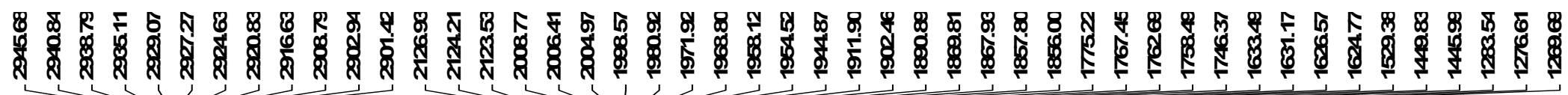
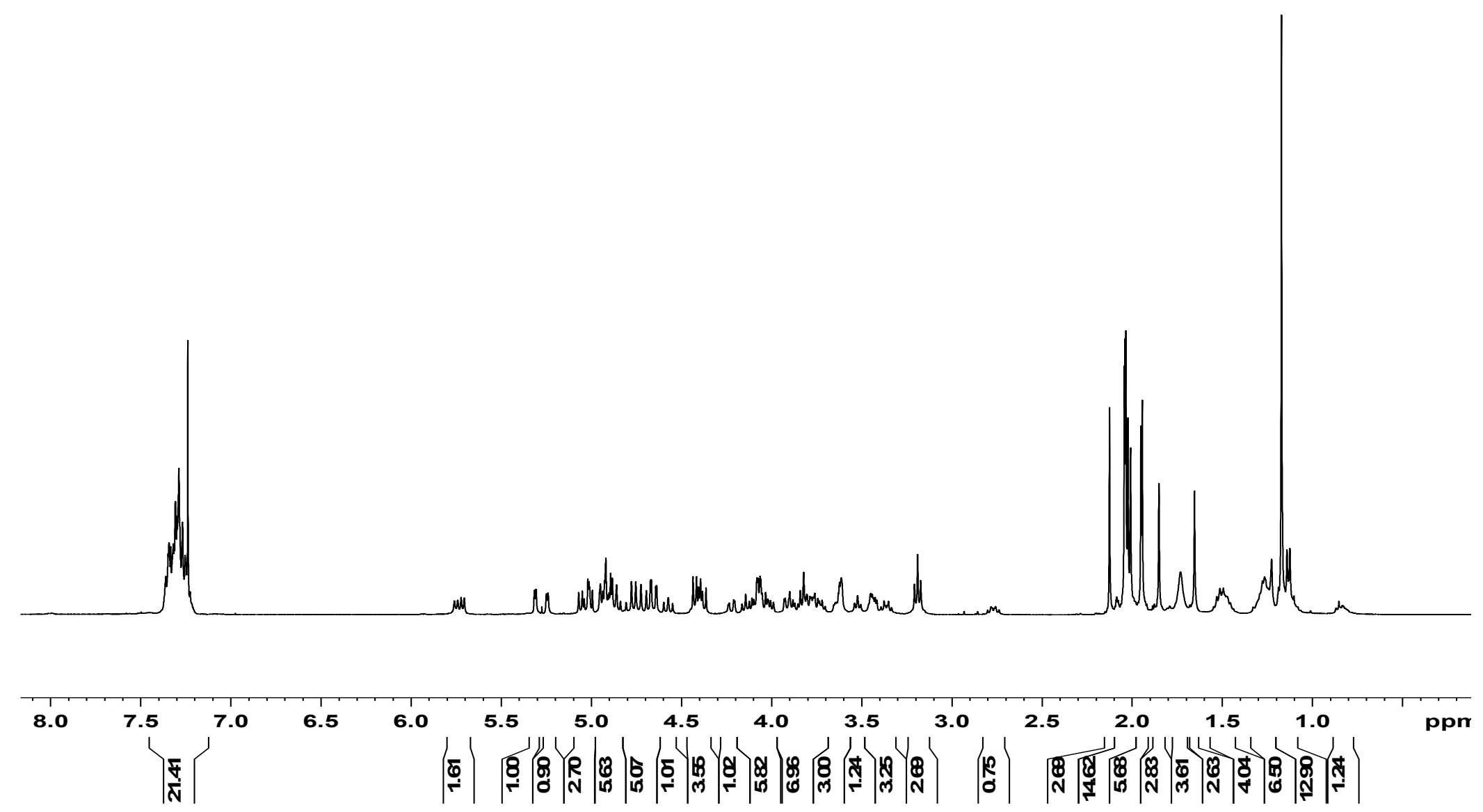
Compound 26, $101 \mathrm{MHz}, 296 \mathrm{~K}, \mathrm{CDCl}_{3}$

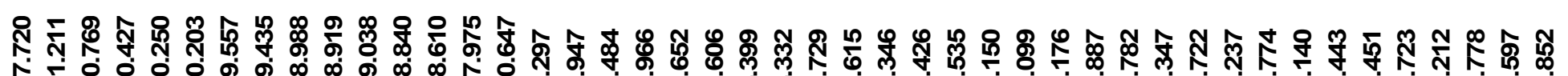
E

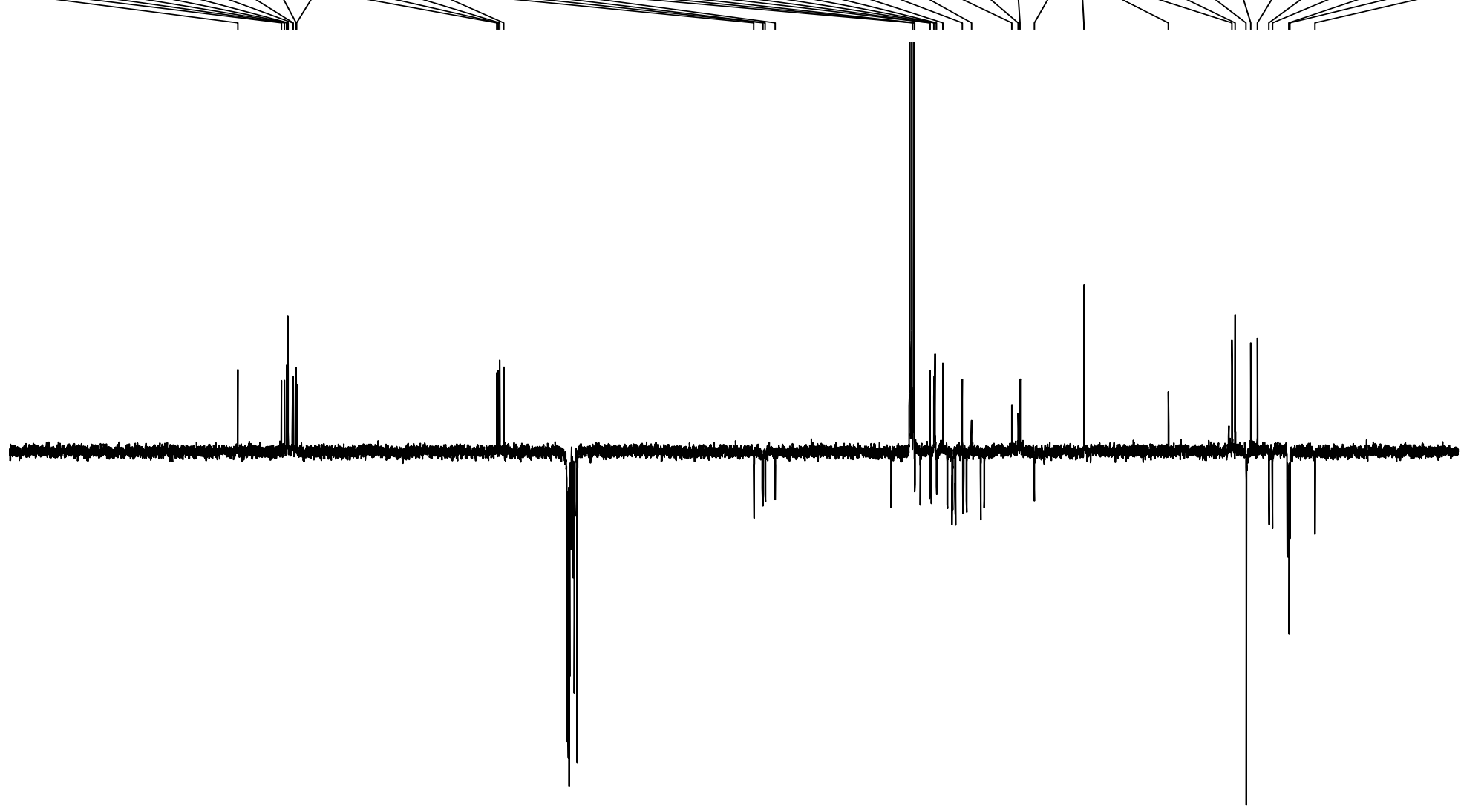

\begin{tabular}{|cccc|ccc|c|c|c|c|}
\hline 200 & 180 & 160 & 140 & 120 & 100 & 80 & 60 & 40 & 20 & ppm
\end{tabular}


Compound 2, $600 \mathrm{MHz}, 296 \mathrm{~K}, \mathrm{D}_{2} \mathrm{O}$

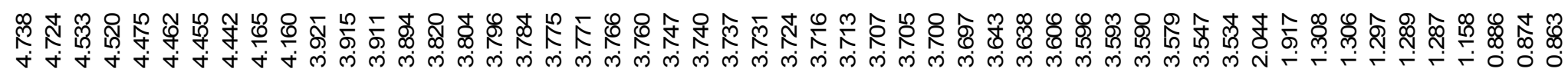

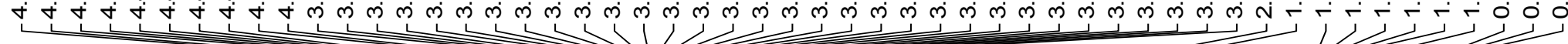

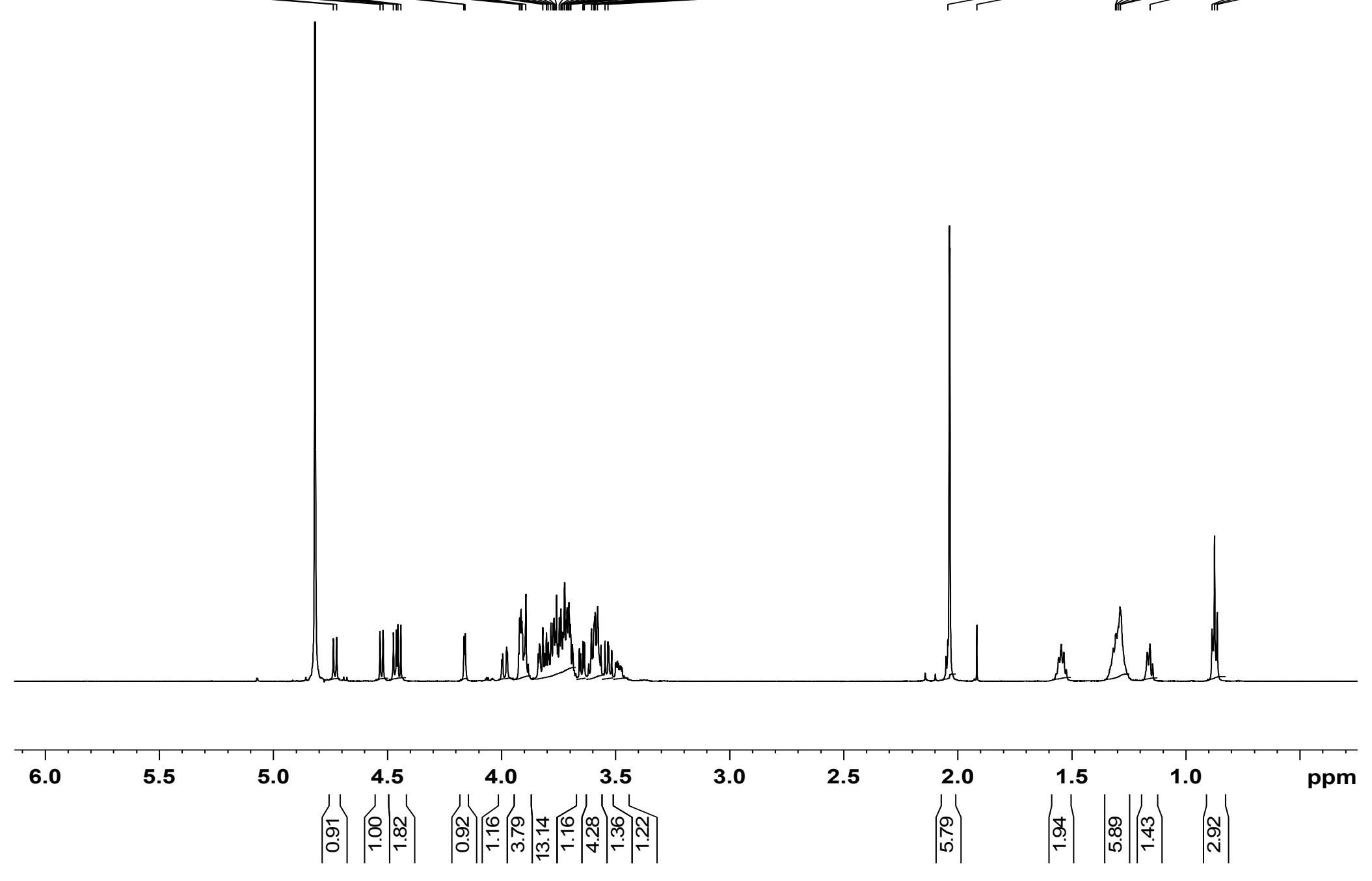


Compound 2, $151 \mathrm{MHz}, 296 \mathrm{~K}, \mathrm{D}_{2} \mathrm{O}$

$$
\text { 点 }
$$

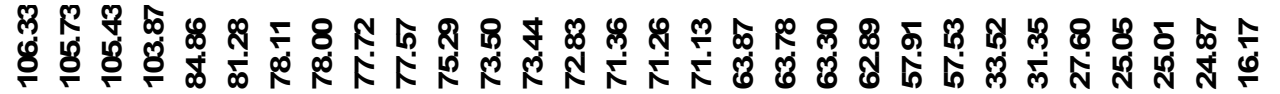

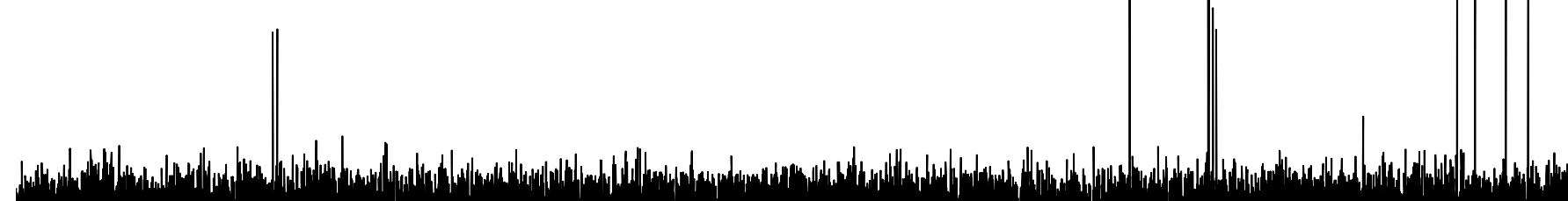

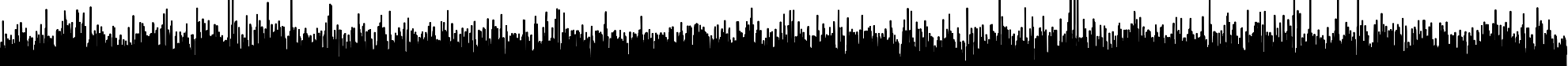

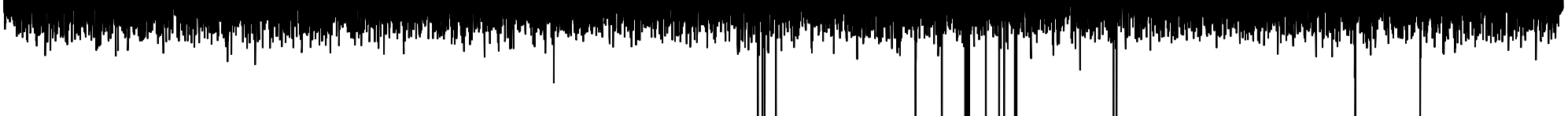

$\begin{array}{llllllllllllllllllllllll}200 & 190 & 180 & 170 & 160 & 150 & 140 & 130 & 120 & 110 & 100 & 90 & 80 & 70 & 60 & 50 & 40 & 30 & 20 & 10 & \text { ppm }\end{array}$ 
Compound 3, $600 \mathrm{MHz}, 295 \mathrm{~K}, \mathrm{D}_{2} \mathrm{O}$

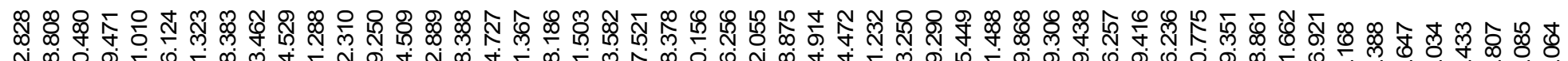

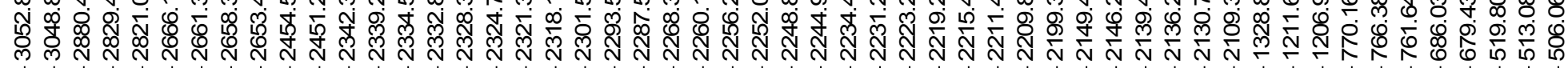

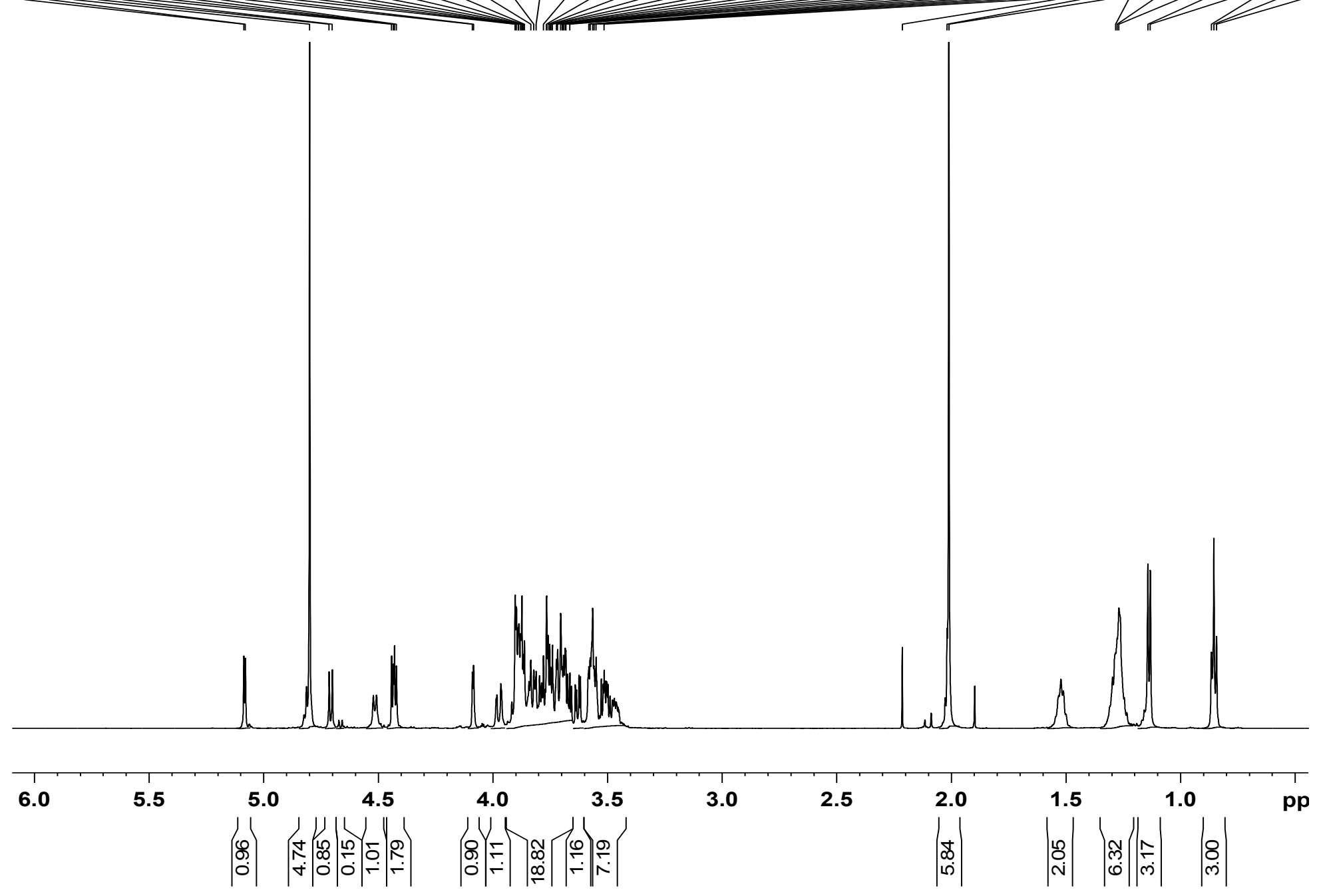


Compound 3, $151 \mathrm{MHz}, 295 \mathrm{~K}, \mathrm{D}_{2} \mathrm{O}$

象员

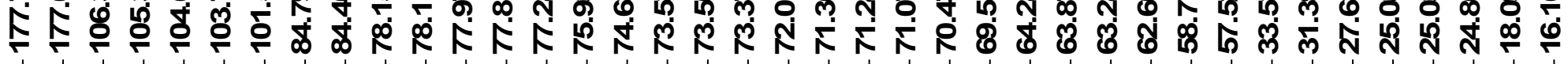

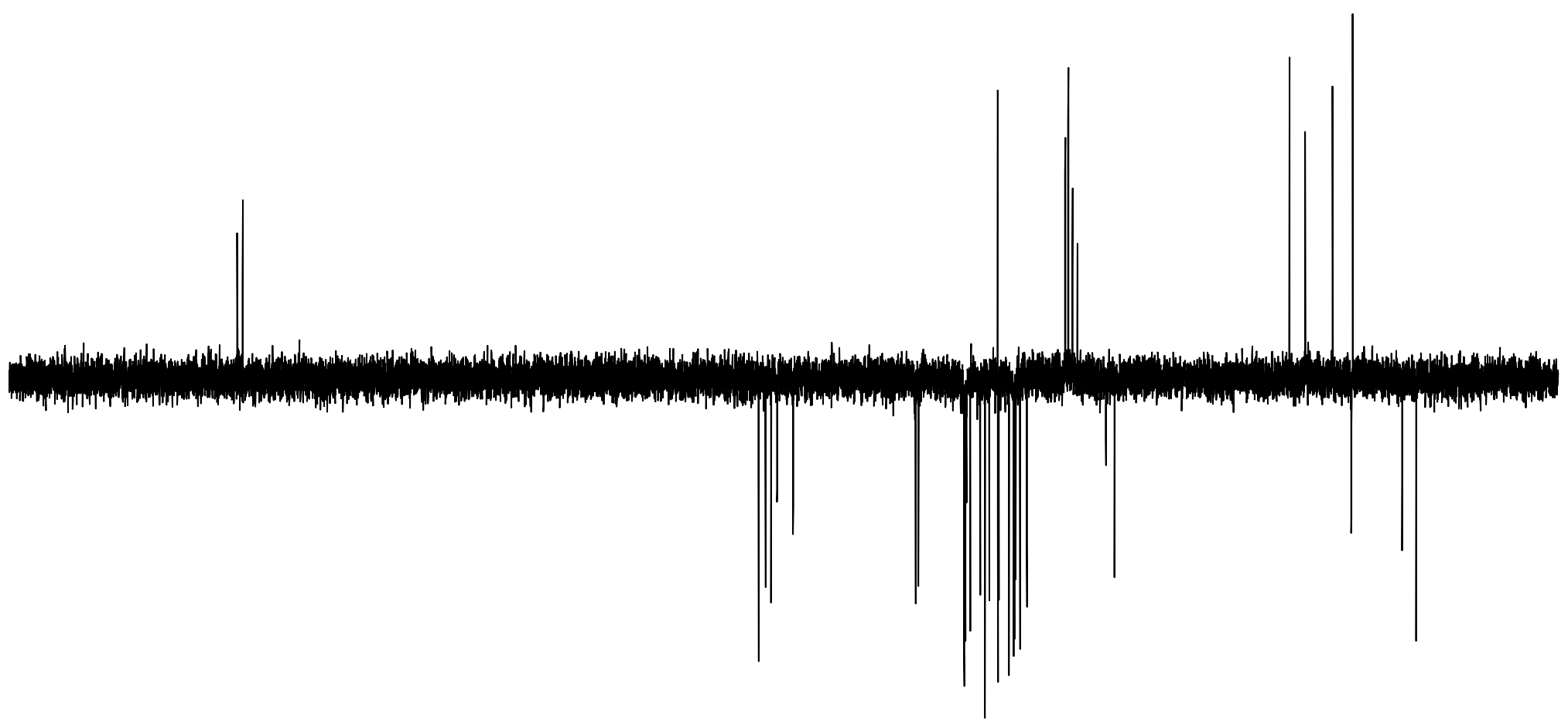

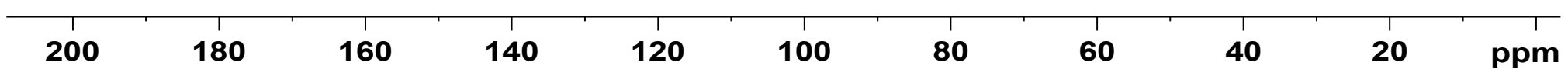




\section{Compound 4, $600 \mathrm{MHz}, 295 \mathrm{~K}, \mathrm{D}_{2} \mathrm{O}$}

\section{C)

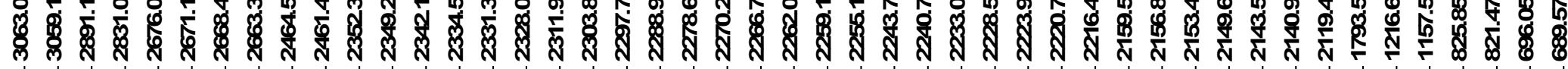

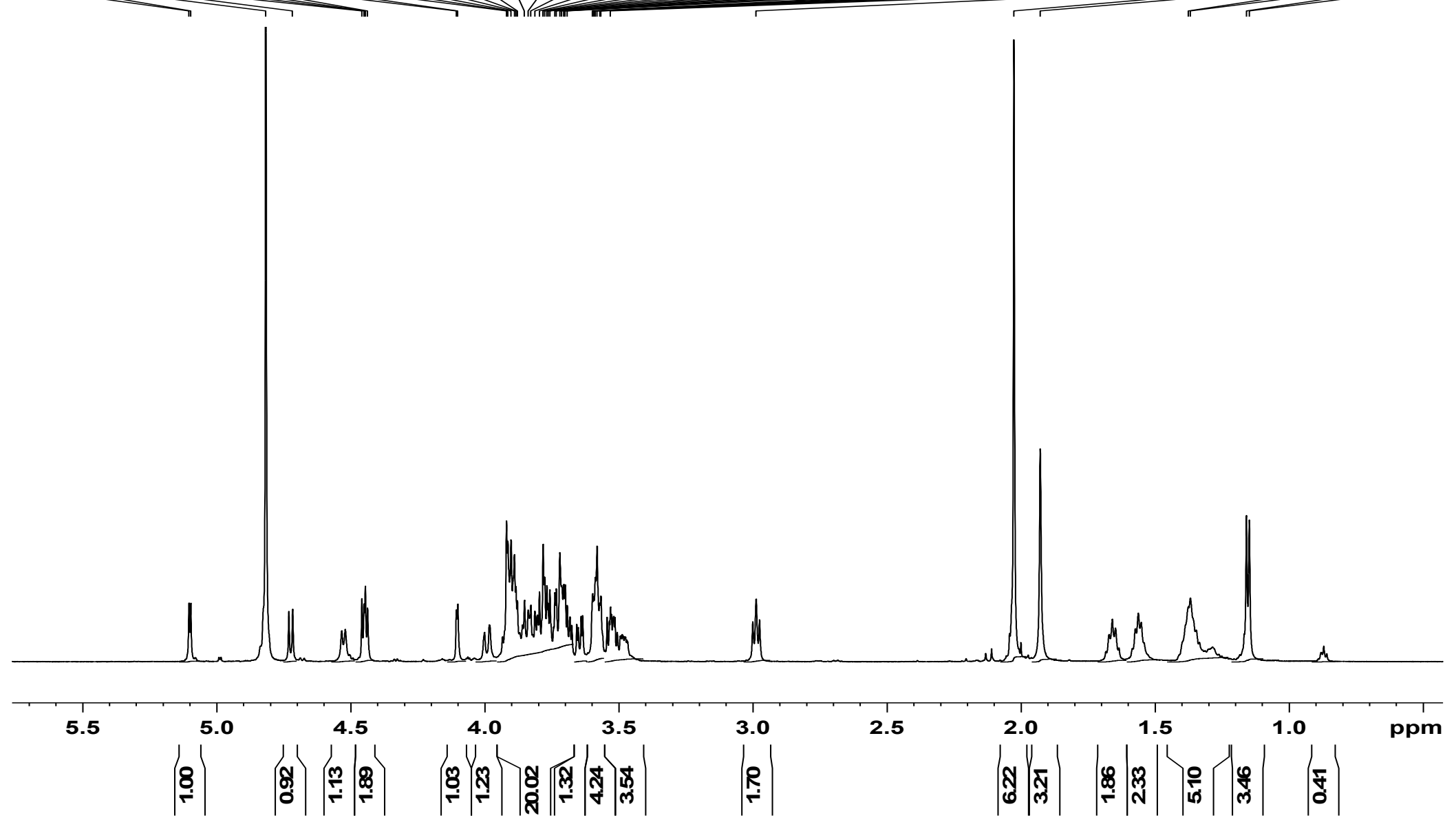


Compound 4, $151 \mathrm{MHz}, 295 \mathrm{~K}, \mathrm{D}_{2} \mathrm{O}$

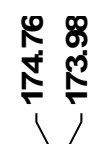

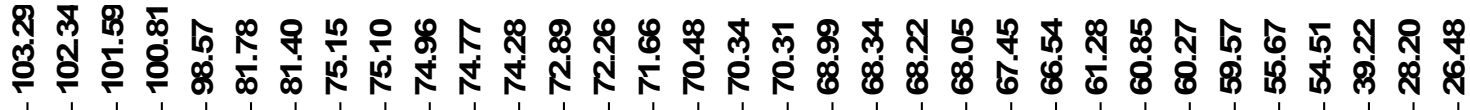

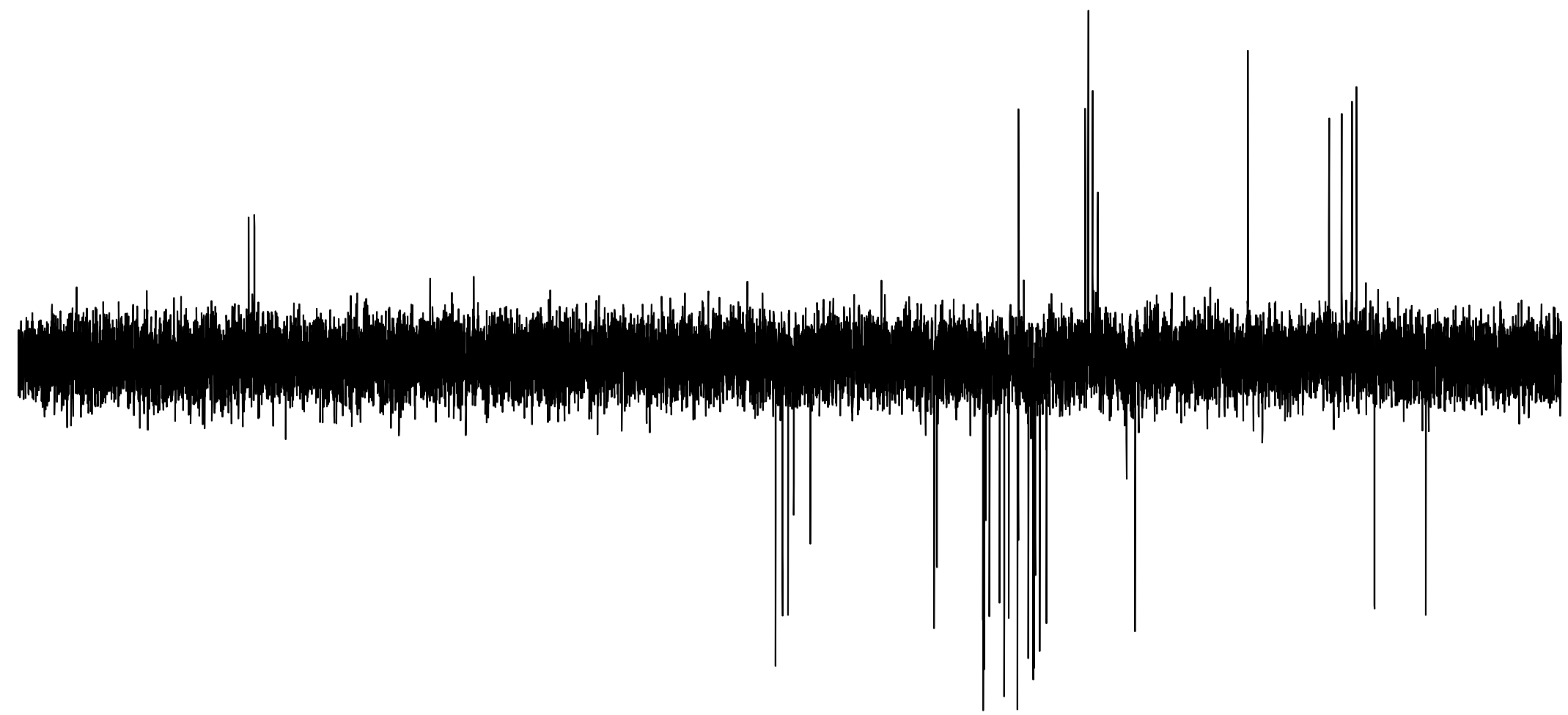

$\begin{array}{lllllllllllllllllllll}200 & 190 & 180 & 170 & 160 & 150 & 140 & 130 & 120 & 110 & 100 & 90 & 80 & 70 & 60 & 50 & 40 & 30 & 20 & 10 & \text { ppm }\end{array}$ 